\title{
THE GROUP OF MOTIONS OF AN EINSTEIN SPACE*
}

BY

\author{
JOHN EIESLAND
}

\section{IN'TRODUCTION}

The question to what extent the general Finstein space is determined by its group of motions seems to be of interest from a physical as well as a geometric standpoint.

In what follows we have discussed the problem of determining the group of motions in a given Riemannian $n$-space and its converse (Killing's equations). The assumption is then made that an Einstein space whose linear element is

$$
-d s^{2}=\sum_{1}^{3} g_{i k} d x_{i} d x_{k}-\sum_{0}^{3} g_{i 0} d x_{i} d x_{0}, \quad x_{0}=t,
$$

shall admit the group of "rotations"

$$
G_{3}: \quad x^{i} \frac{\partial f}{\partial x_{k}}-x_{k} \frac{\partial f}{\partial x_{i}} \quad(i, k=1,2,3)
$$

and the following theorem is proved:

$A$ necessary and sufficient condition that the space (a) shall be reducible. to the form

$$
-d s^{2}=\varphi_{2} d r^{2}+\varphi_{3}\left(d \theta^{2}+\sin ^{2} \theta d \varphi^{2}\right)-\varphi_{1} d t^{2},
$$

$\varphi_{1}, \varphi_{2}$ and $\varphi_{3}$ being arbitrary functions of $r$ and $t$, is that it shall admit the group $G_{3}$ as a complete group of motions.t

It may further be required that (b) admit a one-parameter group

$$
\xi_{0} \frac{\partial f}{\partial t}+\xi_{1} \frac{\partial f}{\partial r}
$$

where $\xi_{0}$ and $\xi_{1}$ are functions of $r$ and $t$. The necessary and sufficient conditions that this shall be the case are found to be

\footnotetext{
** Presented to the Society, March 26, 1921.

$\dagger$ This theorem has generally been taken for granted by writers on relativity.
} 


$$
\begin{aligned}
\varphi_{1} \varphi_{3} G_{1}^{0} & =\Psi^{\prime}\left(\varphi_{3}\right) \frac{\partial \varphi_{3}}{\partial t} \frac{\partial \varphi_{3}}{\partial r} \\
\varphi_{1} \varphi_{2} \varphi_{3}\left(G_{1}^{1}-G_{0}^{0}\right) & =+2 \boldsymbol{s}^{\prime}\left(\varphi_{3}\right)\left[\varphi_{1} \frac{\partial \varphi_{3}}{\partial r}+\varphi_{2} \frac{\partial \varphi_{3}}{\partial t}\right],
\end{aligned}
$$

( $\Psi$ an arbitrary function of $\varphi_{3}$ or a constant). It is then shown that if these conditions are satisfied, the space $(b)$ may be reduced to the static form.

Special forms of static spaces are then considered with special reference to their group properties and the principal curvature of their sub-spaces

$$
S_{3}: t=0 ; \quad S_{3}^{1}: \varphi=0 ; \quad S_{3}^{2}: \quad \theta=0
$$

The question of the class of the quadratic form $(b)$ is then taken up, and it is proved that a necessary and sufficient condition that (b) shall be of class 1 is

$$
(02,02)(13,13)=-=(01,01)(23,23)+(12,02)(13,03) \text {. }
$$

The general space $(b)$ can therefore be immersed in a flat 6-space, and, if $(c)$ is satisfied, in a flat 5-space.

It is also proved that if a general space (a) admits any one of the abelian groups

$$
\frac{\partial f}{\partial x_{0}} ; \quad \frac{\partial f}{\partial x_{0}}, \frac{\partial f}{\partial x_{3}} ; \quad \frac{\partial f}{\partial x_{0}}, \frac{\partial f}{\partial x_{3}}, \frac{\partial f}{\partial x_{2}}
$$

as complete group of motions, it is of the fifth, third, and second class respectively. Among these spaces is found Weyl's static and cylindrical space admitting an abelian $G_{2}$.

1. The general differential quadratic form. Let there be given a general differential quadratic form

$$
d s^{2}=\sum_{1}^{n} a_{i k} d x_{i} d x_{k}
$$

which may be interpreted as the linear element of a curved space $S_{n}$ of $n$ dimensions. This space is said to admit of a group of rigid motions, if there exists a group of transformations

$$
x_{i}^{\prime}=f_{i}\left(x_{1}, x_{2}, \cdots, x_{n}, a_{1}, a_{2}, \cdots, a_{r}\right) \quad(i=1,2, \cdots, n),
$$

which will carry the form (1) into the form

$$
d s^{2}=\sum_{1}^{n} a_{i k}^{\prime} d x_{i}^{\prime} d x_{k}^{\prime}
$$


such that the coefficients $a_{i k}^{\prime}$ are the same functions of $x_{i}^{\prime}$ as the $a_{i k}$ 's are of $x_{i}$. If the coefficients $a_{i k}$ are perfectly general, no such group exists.

In order that a given form (1) shall admit a group*

$$
U f^{\prime}=\xi_{1} \frac{\partial f}{\partial x_{1}}+\xi_{2} \frac{\partial f}{\partial x_{2}}+\cdots+\xi_{n} \frac{\partial f}{\partial x_{n}},
$$

the $\xi$ 's must satisfy the so-called Killing's equations,

$$
\sum_{1}^{n} \xi_{\lambda} \frac{\partial a_{i k}}{\partial x_{\lambda}}+\sum_{1}^{n} a_{i \lambda} \frac{\partial \xi_{\lambda}}{\partial x_{k}}+\sum_{1}^{n} a_{k \lambda} \frac{\partial \xi_{\lambda}}{\partial x_{i}}=0 \quad(i, k=1,2, \cdots, n),
$$

the integration of which will determine the $\xi$ 's as functions of $x_{i}$ and $r$ constants of integration. The maximum group has $r=n(n+1) / 2$ parameters, in which case the space $S_{n}$ has a constant Riemannian curvature. Bianchit gives Killing's equations another form,

$$
\frac{\partial \eta_{i}}{\partial x_{l c}}+\frac{\partial \eta_{l}}{\partial x_{i}}=2 \sum_{\lambda}^{1 \cdots n}\left\{\begin{array}{c}
i k \\
\lambda
\end{array}\right\} \eta_{\lambda}, \quad \eta_{i}=\sum_{\lambda}^{1 \cdots n} a_{i \lambda} \xi_{\lambda} \quad\left(i, l_{i}=1,2, \cdots, n\right),
$$

where $\left\{\begin{array}{c}i k \mid \\ \lambda \mid\end{array}\right.$ are the usual Christoffel symbols. All the second derivatives obtained from these equations can be expressed linearly and homogeneously in terms of the $\eta$ 's and their first derivatives. We thus obtain the system

$$
\begin{aligned}
\frac{\partial^{2} \eta_{i}}{\partial x_{k} \partial x_{l}}=\frac{\partial}{\partial x_{l}} \sum_{\lambda}^{1 \cdots n}\left\{\begin{array}{c}
i k \\
\lambda
\end{array}\right\} \eta_{j}+\frac{\partial}{\partial x_{k}} \sum_{i}^{1 \cdots n}\left\{\begin{array}{c}
i l \\
\lambda
\end{array}\right\}_{\eta_{\lambda}}-\frac{\partial}{\partial x_{i}} \sum_{\lambda}^{1 \cdots n}\left\{\begin{array}{c}
k l \\
\lambda
\end{array}\right\}_{\eta_{i}} \\
(i, k, l=1,2, \cdots, n) .
\end{aligned}
$$

If the systems (4) and (5) are completely integrable, the group has $r=n(n+1) / 2$ parameters. If $r<n(n+1) / 2$, the system is not complete. If therefore we form the conditions of integrability, we find new relations between the $\eta$ 's and their first derivatives which must be added to the system (4). Continuing in this way we shall eventually arrive at the complete Lie-Mayer system defining the group.

2. Let us suppose that a space $S_{n}$ admits at least a one-parameter group $G_{1}$. By proper choice of variables this group may always be reduced

* It is clear that if (1) is invariant under the $\infty^{r}$ finite transformations of the group (2) it is also invariant under the corresponding $r$ infinitesimal transformations of the group. For proof of the converse see L. Bianchi, Lezioni sulla Teoria dei Gruppi Continui di Transformazioni, Pisa, 1918, pp. 493-495.

$\dagger$ L. Bianchi, loc. cit., pp. 502-503. 
to the form $\partial f / \partial x_{1}$. If therefore we put $\xi_{1}=1, \xi_{2}=\xi_{3}=\ldots=\xi_{n}=0$ in Killing's equations (3), we find

$$
\frac{\partial a_{i k}}{\partial x_{1}}=0
$$

which means that the coefficients $a_{i k}$ do not contain $x_{1}$. Hence, if a space $S_{n}$ admits a one-parameter group of motions, its linear element can always be put in the form

$$
d s^{2}=\sum_{0}^{3} a_{i k} d x_{i} d x_{k}
$$

where the coefficients $a_{i k}$ do not contain $x_{1}{ }^{*}$

Suppose further that the group $G_{1}$ is such that the infinitesimal motion at every point of $S_{n}$ has a constant amplitude. A motion of this kind corresponds to a translation in ordinary euclidean space (Schiebung). Since we have $\delta x_{i}=\xi_{i} \delta t$, the condition to be satisfied, in addition to those of equations (3), is

$$
\frac{\delta s^{2}}{\delta t^{2}}=\sum_{0}^{3} a_{i k} \xi_{i} \xi_{k}=\text { const. }
$$

the $\xi$ 's are therefore the constants of direction at any point in $S_{n}$. If we reduce $d s^{2}$ to the form (6) and apply (7) we find $a_{11}=$ const. But the condition $a_{11}=$ const. is the condition that the line $x_{1}$ shall be a geodesic in $S_{n} . t$ We have therefore the

Theorem I. An infinitesimal motion is a translation if, and only if; the trajectories of the group $G_{1}$ generated by it are geodesic lines in $S_{n}$.

Any finite translation carries all the points of space the same geodesic distance from their original positions.

We shall state the following proposition, the proof of which we shall omit:

If the space $S_{n}$ admits a translation, any spread formed by $\infty^{1}$ trajectories of the motion is of zero curvature.

3. The space of a four-dimensional metric field. After these preliminaries which are largely restatements of well known theorems we shall proceed to study the four-dimensional metric field of Einstein's relativity theory, with a special view to its group-theoretical properties.

* 'The converse is also true: If the linear element of $S_{n}$ can be put in the form (6), the space admits at least a one-parameter group of motions.

$\dagger$ L. Bianchi, loc. cit., p. 500 .

† Loc. cit., p. 500 . 
Consider the quadratic form

$$
d s^{2}=\sum_{0}^{3} g_{i k} d x_{i} d x_{k},
$$

which in Einstein's relativity theory contains the metrical relations of time and physical space. Let $x_{0}=t, t$ being interpreted as time, and let $x_{1}, x_{2}, x_{3}$ be the coördinates of a space such that its linear element

We may therefore put

$$
d s_{0}^{2}=-\left(d s^{2}\right)_{d x_{0}=0} \text {. }
$$

$$
d s_{0}^{2}=-\sum_{1}^{3} g_{i k} d x_{i} d x_{k}=\sum_{1}^{3} a_{i k} d x_{i} d x_{k},
$$

and we shall assume moreover that this form is positive and definite. The general quadratic form (9) may therefore be written

$$
d s^{2}=g_{00} d t^{2}+\sum_{0}^{3} g_{0 i} d x_{i} d t-\sum_{1}^{8} a_{i k} d x_{i} d x_{k}
$$

which is indefinite, the index of inertia being 3. $g_{00}$ may be interpreted as a velocity; for, if $t$ only varies, we have $d s^{2} / d t^{2}=g_{00}=V^{2}$ so that $\sqrt{g_{00}}=V$ has the dimension of velocity.

Let us assume that the coefficients $g_{00}, g_{i 0}$ and $a_{i k}$ do not contain $t$. By Theorem I this means that (10) admits at least a one-parameter group of motions, namely

$$
U f=\frac{\partial f}{\partial t}
$$

the invariant spreads of which are the 3-spreads $t=$ const. A space of this kind we shall call with I.evi-Civita a stationary space, so that we have the

THEOREM II. A necessary and sufficient condition that a general Einstein space (10) shall be stationary is that it shall admit the group (11). This motion is a "translation" if, and only if, $g_{00}$ is a constant (Theorem I).

The path-curves of the transformation (11) are not in general geodesics in $S_{4}$. Only when $g_{00}$ is a constant will this be the case, and (10) may be reduced to the geodesic form

$$
d s^{2}=c^{2} d t^{2}-\sum_{1}^{3} a_{i l i} d x_{i} d x_{k}
$$

in which the coefficients $g_{i 0}$ are absent and the new coefficients $a_{i k}$ do not contain $t$ as before. Since $\infty^{1}$ paths-curves of the "translation" will 
form a spread of zero curvature, the space (12) may be described as "cylindrical".*

We shall, however, assume that the space (10) is general, the coefficients $g_{i k}$ being functions of $x_{i}, i=0,1,2,3$. By means of the transformation

$$
x_{0}^{\prime}=x_{0}, \quad x_{i}^{\prime}=x_{i}^{\prime}\left(x_{0}, x_{1}, x_{2}, x_{3}\right) \quad(i=1,2,3)
$$

we may remove the coefficients $g_{i 0}$ in (10); in fact, it will be necessary and sufficient that the functions $x_{i}^{\prime}$ shall be solutions of the differential equation

$$
\nabla\left(x_{0}, \theta\right)=\sum_{0}^{3} j^{i 0} \frac{\partial \theta}{\partial x_{i}}=0
$$

$\nabla\left(x_{0}, x_{i}^{\prime}\right)=0$ being the conditions that the space $x_{1}^{\prime}, x_{2}^{\prime}, x_{3}^{\prime}$ shall be orthogonal to the coördinate line $x_{0}^{\prime}$. The space (10) has now the form

$$
d s^{2}=g_{00} d x_{0}^{2}-\sum_{1}^{3} a_{i k} d x_{i} d x_{k} .
$$

Let us suppose that this space admits a group $G$ and let the general nature of this group be left arbitrary for the time being, except that it does not operate on $x_{0}=t$, i. e., it is a group of the sub-space $x_{0}=$ const. We write then

The equations (3) are

$$
U f=\xi_{1} \frac{\partial f}{\partial x_{1}}+\xi_{2} \frac{\partial f}{\partial x_{2}}+\xi_{3} \frac{\partial f}{\partial x_{3}} .
$$

$$
\begin{gathered}
\sum_{1}^{3} \xi_{i} \frac{\partial g_{00}}{\partial x_{\lambda}}+2 \sum_{1}^{3} g_{0 \lambda} \frac{\partial \xi_{\lambda}}{\partial x_{0}}=0, \quad \sum_{1}^{3} \xi_{\lambda} \frac{\partial a_{11}}{\partial x_{\lambda}}+2 \sum_{1}^{3} a_{1 \lambda} \frac{\partial \xi_{\lambda}}{\partial x_{1}}=0 \\
\sum_{1}^{3} \xi_{\lambda} \frac{\partial a_{22}}{\partial x_{\lambda}}+2 \sum_{1}^{3} a_{2 \lambda} \frac{\partial \xi_{\lambda}}{\partial x_{2}}=0, \quad \sum_{1}^{3} \xi_{\lambda} \frac{\partial a_{33}}{\partial x_{\lambda}}+2 \sum_{1}^{3} a_{3 \lambda} \frac{\partial \xi_{\lambda}}{\partial x_{3}}=0 \\
\sum_{1}^{3} \xi_{i} \frac{\partial a_{i k}}{\partial x_{\lambda}}+\sum_{1}^{3} a_{i \lambda} \frac{\partial \xi_{\lambda}}{\partial x_{\lambda}}+\sum_{1}^{3} a_{k \lambda} \frac{\partial \xi_{\lambda}}{\partial x_{\lambda}}=0 \\
\sum_{1}^{3} a_{1 \lambda} \frac{\partial \xi_{\lambda}}{\partial x_{0}}=0, \quad \sum_{1}^{3} a_{2 \lambda} \frac{\partial \xi_{\lambda}}{\partial x_{0}}=0, \quad \sum_{1}^{3} a_{3 \lambda} \frac{\partial \xi_{\lambda}}{\partial x_{0}}=0 .
\end{gathered}
$$

* The term "static" instead of "stationary" has been used by G. D. Birkhoff in a recent publication, Relativity and Modern Physics (Cambridge, Harvard University Press, 1923). If we consider the hydrodynamic analogy, it would seem that the term "stationary" is a better term. We do not speak of a "static" motion in hydrodynamics, when a stationary or permanent motion is meant. The term "static" field is used by T. Levi-Civita to denote a stationary field in which the coefficients $g_{i 0}$ are absent. See T. Levi-Civita, La Teoria di Einstein e il Principio di Fermat, Il N u o vo Cimento, ser. 6, vol. 16 (1918), pp. 105-114. 
Since the determinant $\left|a_{i k}\right|$ cannot vanish, the last three equations show that the $\xi$ 's are independent of $x_{0}$. The first of equations (15) becomes

$$
\sum_{1}^{8} \xi_{\lambda} \frac{\partial g_{00}}{\partial x_{\lambda}}=0
$$

which means that $g_{00}$ is of the form $g_{00}\left(\varphi, x_{0}\right)$ where $\varphi$ is an invariant of the group $G$, or else a function of $x_{0}$ alone, in which case $g_{00}$ may be reduced to a constant. In the first case, since $G$ does not involve $x_{0}, g_{00}$ is itself an invariant of the group.

(a) $g_{00}$ an invariant of $G$. $G$ must be an intransitive group considered as belonging to $S_{4}$. But since $G$ does not contain $x_{0}$, nor operate on $x_{0}$, it must be a group of motions in $S_{3}$; this is also clear when we consider that the remaining equations in the system (15) are Killing's equations corresponding to the space $S_{3}$. It should be noted that this does not prevent $G$ from being a subgroup of a transitive group $\bar{G}$ of motions in $S_{3}$, but $G$ will not belong to $S_{4}$ unless $g_{00}$ is a function of $x_{0}$ alone, or a constant.

(b) $g_{00}=$ const. In this case $G$ may be any group of motions in $S_{3}$, transitive or intransitive; it may even be the maximum group $G_{6}$ in which case $S_{3}$ is a space of constant positive or zero curvature. If $G$ is a transitive group in $S_{3}$, it can belong to $S_{4}$ if, and only if, $g_{00}$ is a function of $x_{0}$ alone or a constant. We shall state these results in the following

Theorem III. If the space whose linear element is

$$
d s^{2}=g_{00} d x_{0}^{2}-\sum_{1}^{8} a_{i k} d x_{i} d x_{k}
$$

admits a group of the form

$$
U f=\xi_{1} \frac{\partial f}{\partial x_{1}}+\xi_{2} \frac{\partial f}{\partial x_{2}}+\xi_{3} \frac{\partial f}{\partial x_{3}},
$$

the $\xi$ 's are independent of $x_{0}$, and the group belongs also to the subspace $S_{3} . g_{00}$ is either an invaliant of the group, or a function of $x_{0}$ alone. In the first case, the group is intransitive. In the second case, $g_{00}$ may by a transformation be reduced to a constant, and the group is either transitive or intransitive. $A$ transitive group in $S_{3}$ belongs to $S_{4}$ if, and only if, $g_{00}$ is a constant.

4. The group of "rotations" in $S_{3}{ }^{*}$ We shall suppose that $S_{4}$ admits the intransitive group of "rotations" about the origin in $S_{3}$, viz.

$$
x_{1} \frac{\partial f}{\partial x_{2}}-x_{2} \frac{\partial f}{\partial x_{1}}, x_{2} \frac{\partial f}{\partial x_{3}}-x_{3} \frac{\partial f}{\partial x_{2}}, x_{3} \frac{\partial f}{\partial x_{1}}-x_{1} \frac{\partial f}{\partial x_{3}} .
$$

* By a "group of rotations" we mean here a 3-parameter group in the variables $x_{1}, x_{2}, x_{8}$ having the invariant $x_{1}^{2}+x_{2}^{2}+x_{3}^{2} ; x_{1}, x_{2}, x_{3}$ are not cartesian coördinates. 
The sub-space $S_{3}$ is then said to be centro-symmetric. If in (15) we introduce in succession the following values of the $\xi$ 's,

$$
\begin{array}{lll}
\xi_{1}=-x_{2}, & \xi_{2}=x_{1}, & \xi_{3}=0, \\
\xi_{1}=0, & \xi_{2}=-x_{3}, & \xi_{3}=x_{2}, \\
\xi_{1}=x_{3}, & \xi_{2}=0, & \xi_{3}=-x_{1},
\end{array}
$$

we obtain a system of equations for determining the quantities $g_{00}$ and $a_{i k}$ :

(a)

$$
x_{1} \frac{\partial g_{00}}{\partial x_{2}}-x_{2} \frac{\partial g_{00}}{\partial x_{1}}=0, \quad x_{2} \frac{\partial g_{00}}{\partial x_{3}}-x_{3} \frac{\partial g_{00}}{\partial x_{2}}=0 \text {, }
$$

$$
x_{3} \frac{\partial g_{00}}{\partial x_{1}}-x_{1} \frac{\partial g_{00}}{\partial x_{3}}=0
$$

$$
\begin{gathered}
x_{1} \frac{\partial a_{11}}{\partial x_{2}}-x_{2} \frac{\partial a_{11}}{\partial x_{1}}+2 a_{12}=0, \quad x_{2} \frac{\partial a_{11}}{\partial x_{3}}-x_{3} \frac{\partial a_{11}}{\partial x_{2}}=0, \\
x_{3} \frac{\partial a_{11}}{\partial x_{1}}-x_{1} \frac{\partial a_{11}}{\partial x_{3}}-2 a_{13}=0, \\
x_{1} \frac{\partial a_{22}}{\partial x_{2}}-x_{2} \frac{\partial a_{22}}{\partial x_{1}}-2 a_{12}=0, \quad x_{2} \frac{\partial a_{22}}{\partial x_{3}}-x_{3} \frac{\partial a_{22}}{\partial x_{2}}+2 a_{23}=0, \\
x_{3} \frac{\partial a_{22}}{\partial x_{1}}-x_{1} \frac{\partial a_{22}}{\partial x_{3}}=0, \\
x_{1} \frac{\partial a_{33}}{\partial x_{2}}-x_{2} \frac{\partial a_{33}}{\partial x_{1}}=0, \quad x_{2} \frac{\partial a_{33}}{\partial x_{3}}-x_{3} \frac{\partial a_{33}}{\partial x_{2}}-2 a_{23}=0, \\
x_{3} \frac{\partial a_{33}}{\partial x_{1}}-x_{1} \frac{\partial a_{33}}{\partial x_{3}}+2 a_{13}=0,
\end{gathered}
$$

(b)

$$
\begin{gathered}
x_{1} \frac{\partial a_{12}}{\partial x_{2}}-x_{2} \frac{\partial a_{12}}{\partial x_{1}}-a_{11}+a_{z 2}=0, \quad x_{2} \frac{\partial a_{12}}{\partial x_{3}}-x_{3} \frac{\partial a_{12}}{\partial x_{2}}+a_{13}=0, \\
x_{3} \frac{\partial a_{12}}{\partial x_{1}}-x_{1} \frac{\partial a_{12}}{\partial x_{3}}-a_{23}=0 \\
x_{1} \frac{\partial a_{13}}{\partial x_{2}}-x_{2} \frac{\partial a_{13}}{\partial x_{1}}+a_{23}=0, \quad x_{2} \frac{\partial a_{13}}{\partial x_{3}}-x_{3} \frac{\partial a_{13}}{\partial x_{2}}+a_{13}=0 \\
x_{3} \frac{\partial a_{13}}{\partial x_{1}}-x_{1} \frac{\partial a_{13}}{\partial x_{3}}-a_{33}+a_{11}=0 \\
x_{1} \frac{\partial a_{23}}{\partial x_{2}}-x_{2} \frac{\partial a_{23}}{\partial x_{1}}-a_{13}=0, \quad x_{2} \frac{\partial a_{23}}{\partial x_{3}}-x_{3} \frac{\partial a_{23}}{\partial x_{2}}+a_{22}-a_{33}=0, \\
x_{3} \frac{\partial a_{23}}{\partial x_{1}}-x_{1} \frac{\partial a_{23}}{\partial x_{3}}+a_{12}=0 .
\end{gathered}
$$


If we suppose that $g_{00}$ is not a function of $x_{0}$ alone, the equations $(a)$ express the fact that $g_{00}$ is an invariant of the group $G_{3}$, so that we may put

$$
g_{00}=\varphi_{1}\left(\sqrt{x_{1}^{2}+x_{2}^{2}+x_{8}^{2}}, x_{0}\right) .
$$

We proceed now to integrate $(b)$. By elimination we easily find the following relations:

$$
a_{13}=\frac{x_{3}}{x_{2}} a_{12}, \quad a_{23}=\frac{x_{2}}{x_{1}} a_{12} .
$$

The equations involving $a_{12}, a_{13}, a_{23}$ give, on integrating, keeping account of (18),

$$
a_{12}=x_{1} x_{2} \varphi_{2}, \quad a_{13}=x_{1} x_{3} \varphi_{2}, \quad a_{23}=x_{2} x_{3} \varphi_{2},
$$

$\varsigma_{2}$ being an arbitrary function of $\sqrt{x_{1}^{2}+x_{2}^{2}+x_{3}^{2}}$ and $x_{0}$. We also find the relations

$$
\begin{gathered}
a_{11}-a_{22}=\left(x_{1}^{2}-x_{2}^{2}\right) \varphi_{2}, \quad a_{22}-a_{33}=\left(x_{2}^{2}-x_{3}^{2}\right) \varsigma_{2}, \\
a_{33}-a_{11}=\left(x_{3}^{2}-x_{1}^{2}\right) \varphi_{2} .
\end{gathered}
$$

Integrating the equations in $a_{11}, a_{22}$ and $a_{33}$ we have

$$
a_{11}=\varphi_{3}+x_{1}^{2} \varphi_{2}, \quad a_{22}=\varphi_{3}+x_{2}^{2} \varphi_{2}, \quad a_{33}=\varphi_{3}+x_{3}^{2} \varphi_{2} .
$$

We have thus obtained the following quadratic form,

$d s^{2}=\varphi_{1} d x_{0}^{2}-R^{2} \varphi_{2}\left[\frac{x_{1} d x_{1}+x_{2} d x_{2}+x_{3} d x_{3}}{R}\right]^{2}-\varphi_{3}\left[d x_{1}^{2}+d x_{2}^{2}+d x_{3}^{2}\right]$,

where $R=\sqrt{x_{1}^{2}+x_{2}^{2}+x_{3}^{2}}$. Introducing spherical coördinates

$$
x_{1}=R \sin \theta \cos \varphi, \quad x_{2}=R \sin \theta \sin \varphi, \quad x_{3}=R \cos \theta,
$$

we have, remembering that $\varphi_{2}$ and $\varphi_{3}$ are arbitrary functions of $R$ and $x_{0}$,

$$
d s^{2}=\varphi_{1} d x_{0}^{2}-\left(\varphi_{3}+\varphi_{2}\right) d R^{2}-R^{2} \varphi_{3}\left(d \theta^{2}+\sin ^{2} \theta d g^{2}\right) .
$$

The group $G_{3}$ becomes, on introducing the new variables,

$$
\begin{gathered}
U_{1}=\sin \varphi \frac{\partial f}{\partial \theta}+\cot \theta \cos \varphi \frac{\partial f}{\partial \varphi} \\
U_{2}=\cos \varphi \frac{\partial f}{\partial \theta}-\cot \theta \sin \varphi \frac{\partial f}{\partial \varphi}, \quad U_{3}=\frac{\partial f}{\partial \varphi} .
\end{gathered}
$$


This group is transitive considered as a group in the variables $\theta$ and $\varphi$, and the variable $R$ does not appear, as was to be expected according to a theorem by Fubini.* We have thus proved the

Theorem IV. A necessary and sufficient condition that the space (13) shall be reducible to the form (21) is that it shall admit the group $G_{3}$ as a complete group of motions.

For the purpose of further specialization we shall consider a few invariants that play an important rôle in the classification of 3- and 4-spaces and also in the general relativity theory.

5. The total curvature of $S_{4}$ (curvature scalar) is given by the formula

$$
R=\sum_{h, i}^{0 \ldots 3} g^{h i} R_{h i}, \quad R_{h i}=\sum_{p}^{0 \cdots 3}\{h p, i p\}
$$

If instead of the symbols $\{h p, i p\}$ we introduce the Riemannian symbols $(h p, i q)$, we have

wheret

$$
\{h p, i p\}=\sum_{p, q}^{0 \ldots 3} g^{p q}(h p, i q) \quad R=\sum_{p, q}^{0 \cdots 3} g^{p q}(h p, i q),
$$

$$
\begin{aligned}
(h p, i q)=\frac{1}{2}\left[\frac{\partial^{2} g_{h q}}{\partial x_{i} \partial x_{p}}+\frac{\partial^{2} g_{i p}}{\partial x_{h} \partial x_{q}}\right. & \left.-\frac{\partial^{2} g_{h i}}{\partial x_{p} \partial x_{q}}-\frac{\partial^{2} g_{p q}}{\partial x_{h} \partial x_{i}}\right] \\
& +\sum_{l, m}^{0 \ldots 3} g^{l m}\left(\left[\begin{array}{cc}
h & q \\
m
\end{array}\right]\left[\begin{array}{cc}
i & p \\
l
\end{array}\right]-\left[\begin{array}{cc}
h & i \\
m
\end{array}\right]\left[\begin{array}{ll}
p & q \\
l
\end{array}\right]\right),
\end{aligned}
$$

and the quantities $g^{p q}$ are the co-factors of $g_{p q}$ divided by $g$. We now define the following expressions:

$$
G_{i h}=\frac{1}{2} g_{i h} R-R_{i h},
$$

and, introducing the mixed forms $G_{i}^{h}$, we put

$$
G_{i}^{h}=\sum_{j} g^{h j} G_{j i}
$$

We shall also recall here that for the empty space in an Einstein solar field we must have $R_{i k}=0$, or, what is the same thing, $G_{i}^{h}=0$. Calculating the curvature tensors $R_{i k}$ for the space (21) which we write in the form

$$
-d s^{2}=\varphi_{2} d R^{2}+\varphi_{3}\left(d \theta^{2}+\sin ^{2} \theta d \varphi^{2}\right)-\varphi_{1} d t^{2}
$$

* L. Bianchi, loc. cit., pp. 517-518. See also Fubini's memoir in vol. 3 of Annali di Matematica.

$\dagger$ The non-vanishing Riemannian symbols $(h p, i q)$ are given on p. 238, equations (58). 
we have

$$
\begin{gathered}
g_{00}=-\varphi_{1}, \quad g_{11}=\varphi_{2}, \quad g_{22}=\varphi_{3}, \quad g_{33}=\varphi_{3} \sin ^{2} \theta, \\
g^{11}=\frac{1}{\varphi_{2}}, \quad g^{22}=\frac{1}{\varphi_{3}}, \quad g^{33}=\frac{1}{\varphi_{3} \sin ^{2} \theta}, \quad g^{00}=-\frac{1}{\varphi_{1}} \\
R_{12}=g^{33}(13,23)+g^{00}(10,20), \quad R_{13}=g^{22}(12,32)+g^{00}(10,30), \\
(26 a) R_{23}=g^{11}(21,31)+g^{00}(20,30), \quad R_{10}=g^{22}(12,02)+g^{33}(13,03), \\
R_{20}=g^{11}(21,01)+g^{33}(23,03), \quad R_{30}=g^{11}(31,01)+g^{22}(32,02) ; \\
R_{11}=g^{22}(12,12)+g^{33}(13,13)+g^{00}(10,10), \\
R_{22}=g^{11}(21,21)+g^{33}(23,23)+g^{00}(10,10), \\
R_{33}=g^{11}(31,31)+g^{22}(32,32)+g^{00}(30,30), \\
R_{00}=g^{11}(01,01)+g^{22}(02,02)+g^{33}(30,30) .
\end{gathered}
$$

Calculating the Riemannian symbols $(h p, i q)$ and substituting in these equations we find

$$
\begin{aligned}
R_{12}= & 0, \quad R_{13}=0, \quad R_{23}=0, \quad R_{20}=0, \quad R_{30}=0 \\
R_{10}= & -\frac{1}{\varphi_{3}} \frac{\partial^{2} \varphi_{3}}{\partial r \partial t}+\frac{1}{2 \varphi_{2} \varphi_{3}} \frac{\partial \varphi_{3}}{\partial r} \frac{\partial \varphi_{2}}{\partial t}+\frac{1}{2 \varphi_{3}^{2}} \frac{\partial \varphi_{3}}{\partial r} \frac{\partial \varphi_{3}}{\partial t}+\frac{1}{2 \varphi_{1} \varphi_{3}} \frac{\partial \varphi_{1}}{\partial r} \frac{\partial \varphi_{3}}{\partial t} \\
R_{22}= & \frac{R_{33}}{\sin ^{2} \theta}=-\frac{1}{2 \varphi_{2}} \frac{\partial^{2} \varphi_{3}}{\partial r^{2}}+\frac{1}{4 \varphi_{2}^{2}} \frac{\partial \varphi_{2}}{\partial r} \frac{\partial \varphi_{3}}{\partial r}-\frac{1}{4 \varphi_{1}^{2}} \frac{\partial \varphi_{3}}{\partial t} \frac{\partial \varphi_{1}}{\partial t}+\frac{1}{2 \varphi_{1}} \frac{\partial^{2} \varphi_{3}}{\partial t^{2}} \\
& +\frac{1}{4 \varphi_{1} \varphi_{2}}\left[\frac{\partial \varphi_{2}}{\partial t} \frac{\partial \varphi_{3}}{\partial t}-\frac{\partial \varphi_{1}}{\partial r} \frac{\partial \varphi_{3}}{\partial r}\right]+1, \\
R_{11}= & -\frac{1}{\varphi_{3}} \frac{\partial^{2} \varphi_{3}}{\partial r^{2}}+\frac{1}{2 \varphi_{1}} \frac{\partial^{2} \varphi_{2}}{\partial t^{2}}-\frac{1}{2 \varphi_{1}} \frac{\partial^{2} \varphi_{1}}{\partial r^{2}}+\frac{1}{2 \varphi_{2} \varphi_{3}} \frac{\partial \varphi_{2}}{\partial r} \frac{\partial \varphi_{3}}{\partial r} \\
& +\frac{1}{2 \varphi_{3}^{2}}\left[\frac{\partial \varphi_{3}}{\partial r}\right]^{2}+\frac{1}{2 \varphi_{1} \varphi_{3}} \frac{\partial \varphi_{2}}{\partial t} \frac{\partial \varphi_{3}}{\partial t}+\frac{1}{4 \varphi_{1} \varphi_{2}}\left[\frac{\partial \varphi_{2}}{\partial t}\right]^{2}-\frac{1}{4 \varphi_{1}^{2}}\left[\frac{\partial \varphi_{1}}{\partial r}\right]^{2} \\
& +\frac{1}{4 \varphi_{2}^{2}} \frac{\partial \varphi_{2}}{\partial r} \frac{\partial \varphi_{1}}{\partial t}-\frac{1}{4 \varphi_{1} \varphi_{2}} \frac{\partial \varphi_{2}}{\partial r} \frac{\partial \varphi_{1}}{\partial t}, \\
R_{00}= & -\frac{1}{\varphi_{3}} \frac{\partial^{2} \varphi_{3}}{\partial t^{2}}-\frac{1}{2 \varphi_{2}} \frac{\partial^{2} \varphi_{2}}{\partial t^{2}}+\frac{1}{2 \varphi_{2}} \frac{\partial^{2} \varphi_{1}}{\partial r^{2}}+\frac{1}{2 \varphi_{1} \varphi_{3}} \frac{\partial \varphi_{3}}{\partial t} \frac{\partial \varphi_{1}}{\partial t} \\
& +\frac{1}{2 \varphi_{3}^{2}}\left[\frac{\partial \varphi_{3}}{\partial t}\right]^{2}+\frac{1}{4 \varphi_{2}^{2}}\left[\frac{\partial \varphi_{2}}{\partial t}\right]^{2}+\frac{1}{2 \varphi_{2} \varphi_{3}} \frac{\partial \varphi_{3}}{\partial r} \frac{\partial \varphi_{1}}{\partial r}+\frac{1}{4 \varphi_{1} \varphi_{2}} \frac{\partial \varphi_{2}}{\partial t} \frac{\partial \varphi_{1}}{\partial t} \\
& -\frac{1}{\varphi_{2}^{2}} \frac{\partial \varphi_{2}}{\partial r} \frac{\partial \varphi_{1}}{\partial r}-\frac{1}{4 \varphi_{1} \varphi_{2}}\left[\frac{\partial \varphi_{1}}{\partial r}\right]^{2} ;
\end{aligned}
$$




$$
\begin{gathered}
R=g^{11} R_{11}+g^{22} R_{22}+g^{33} R_{33}+g^{00} R_{00}, \quad G_{i i}=\frac{1}{2} g_{i i} R-R_{i i} \\
G_{i}^{i}=g^{i i} G_{i i}=\frac{1}{2} R-g^{i i} R_{i i}, \quad G_{1}^{0}=g^{00} G_{01}=-g^{00} R_{10} \\
G_{i}^{h}=0, \quad i, h \neq 1,0
\end{gathered}
$$

$$
\begin{aligned}
& G_{1}^{1}=g^{22} g^{33}(23,23)+g^{00} g^{22}(20,20)+g^{33} g^{00}(03,03) \\
& G_{2}^{2}=g^{11} g^{33}(13,13)+g^{11} g^{00}(10,10)+g^{33} g^{00}(03,03) \\
& G_{3}^{3}=g^{11} g^{22}(12,12)+g^{11} g^{00}(10,10)+g^{22} g^{00}(02,02) \\
& G_{0}^{0}=g^{11} g^{22}(12,12)+g^{11} g^{33}(13,13)+g^{22} g^{33}(23,23)
\end{aligned}
$$$$
G_{1}^{1}=\frac{1}{\varphi_{3}}-\frac{1}{4 \varphi_{2} \varphi_{3}^{2}}\left[\frac{\partial \varphi_{3}}{\partial r}\right]^{2}-\frac{1}{4 \varphi_{1} \varphi_{3}^{2}}\left[\frac{\partial \varphi_{3}}{\partial t}\right]^{2}-\frac{1}{2 \varphi_{3} \varphi_{1}^{2}} \frac{\partial \varphi_{3}}{\partial t} \frac{\partial \varphi_{1}}{\partial t}
$$$$
-\frac{1}{2 \varphi_{1} \varphi_{2} \varphi_{3}} \frac{\partial \varphi_{3}}{\partial r} \frac{\partial \varphi_{1}}{\partial r}+\frac{1}{\varphi_{1} \varphi_{3}} \frac{\partial^{2} \varphi_{3}}{\partial t^{2}}
$$$$
G_{0}^{0}=\frac{1}{\varphi_{3}}+\frac{1}{4 \varphi_{2} \varphi_{3}^{2}}\left[\frac{\partial \varphi_{3}}{\partial r}\right]^{2}+\frac{1}{4 \varphi_{1} \varphi_{3}^{2}}\left[\frac{\partial \varphi_{3}}{\partial t}\right]^{2}+\frac{1}{2 \varphi_{2}^{2} \varphi_{3}} \frac{\partial \varphi_{2}}{\partial r} \frac{\partial \varphi_{3}^{\prime}}{\partial r}
$$$$
+\frac{1}{2 \varphi_{1} \varphi_{2} \varphi_{3}} \frac{\partial \varphi_{2}}{\partial t} \frac{\partial \varphi_{3}}{\partial t}-\frac{1}{\varphi_{2} \varphi_{3}} \frac{\partial^{2} \varphi_{3}}{\partial r^{2}}
$$

$$
\begin{aligned}
G_{2}^{2}= & G_{3}^{3}=\frac{1}{2 \varphi_{1} \varphi_{2}} \frac{\partial^{2} \varphi_{2}}{\partial t^{2}}-\frac{1}{2 \varphi_{1} \varphi_{2}} \frac{\partial^{2} \varphi_{1}}{\partial r^{2}}-\frac{1}{2 \varphi_{3} \varphi_{2}} \frac{\partial^{2} \varphi_{3}}{\partial r^{2}} \\
& +\frac{1}{4 \varphi_{2} \varphi_{3}^{2}}\left[\frac{\partial \varphi_{3}}{\partial r}\right]^{2}-\frac{1}{4 \varphi_{1} \varphi_{2}^{2}}\left[\frac{\partial \varphi_{2}}{\partial t}\right]^{2}+\frac{1}{4 \varphi_{2} \varphi_{1}^{2}}\left[\frac{\partial \varphi_{1}}{\partial r}\right]^{2}-\frac{1}{4 \varphi_{1} \varphi_{3}^{2}}\left[\frac{\partial \varphi_{3}}{\partial t}\right]^{2} \\
& +\frac{1}{4 \varphi_{3} \varphi_{2}^{2}} \frac{\partial \varphi_{2}}{\partial r} \frac{\partial \varphi_{3}}{\partial r}-\frac{1}{4 \varphi_{1}^{2} \varphi_{2}} \frac{\partial \varphi_{2}}{\partial t} \frac{\partial \varphi_{1}}{\partial t}-\frac{1}{4 \varphi_{1} \varphi_{2} \varphi_{3}} \frac{\partial \varphi_{3}}{\partial r} \frac{\partial \varphi_{1}}{\partial r} \\
& -\frac{1}{4 \varphi_{1}^{2} \varphi_{3}} \frac{\partial \varphi_{3}}{\partial t} \frac{\partial \varphi_{1}}{\partial t}+\frac{1}{4 \varphi_{1} \varphi_{2} \varphi_{3}} \frac{\partial \varphi_{2}}{\partial t} \frac{\partial \varphi_{3}}{\partial t}+\frac{1}{4 \varphi_{1} \varphi_{2}^{2}} \frac{\partial \varphi_{2}}{\partial r} \frac{\partial \varphi_{1}}{\partial r} \\
& +\frac{1}{2 \varphi_{1} \varphi_{3}} \frac{\partial^{2} \varphi_{3}}{\partial t^{2}}, \\
G_{1}^{0}= & \frac{1}{\varphi_{1}}\left[\frac{1}{\varphi_{3}} \frac{\partial^{2} \varphi_{3}}{\partial r}-\frac{1}{2 \varphi_{2} \varphi_{3}} \frac{\partial \varphi_{2}}{\partial t} \frac{\partial \varphi_{3}}{\partial r}-\frac{1}{2 \varphi_{3}^{2}} \frac{\partial \varphi_{3}}{\partial r} \frac{\partial \varphi_{3}}{\partial t}-\frac{1}{2 \varphi_{1} \varphi_{3}} \frac{\partial \varphi_{1}}{\partial r} \frac{\partial \varphi_{3}}{\partial t}\right] .
\end{aligned}
$$

It is significant that these mixed tensors do not contain any of the variables $\varphi, \theta$, while $R_{33}$ contains $\theta$.

6. We shall now suppose that the space $\left(21^{\prime}\right)$ admits a one-parameter group whose infinitesimal symbol is of the form

$$
G_{1}: \quad U f=\xi_{0} \frac{\partial f}{\partial t}+\xi_{1} \frac{\partial f}{\partial r},
$$


where $\xi_{0}$ and $\xi_{1}$ are functions of $r$ and $t$. In order that this shall be the case, the functions $\varphi_{1}, \varphi_{2}$ and $\varphi_{3}$ must satisfy certain conditions which we shall now proceed to find. The Killing equations (4) are in this case

$$
\begin{aligned}
\xi_{0} \frac{\partial \varphi_{2}}{\partial t}+\xi_{1} \frac{\partial \varphi_{2}}{\partial r}+2 \varphi_{2} \frac{\partial \xi_{1}}{\partial r} & =0, & \xi_{0} \frac{\partial \varphi_{1}}{\partial t}+\xi_{1} \frac{\partial \varphi_{1}}{\partial r}+2 \varphi_{1} \frac{\partial \xi_{0}}{\partial t} & =0, \\
\xi_{0} \frac{\partial \varphi_{3}}{\partial t}+\xi_{1} \frac{\partial \varphi_{3}}{\partial r} & =0, & \varphi_{2} \frac{\partial \xi_{1}}{\partial t}-\varphi_{1} \frac{\partial \xi_{0}}{\partial r} & =0 .
\end{aligned}
$$

The third equation shows that $\varphi_{3}$ must be an invariant of the group or a constant. If $\varphi_{3}$ is not a constant, we put

$$
\xi_{0}=-\lambda \frac{\partial \varphi_{3}}{\partial r}, \quad \xi_{1}=\lambda \frac{\partial \varphi_{3}}{\partial t} .
$$

Substituting the values of $\xi_{0}, \xi_{1}$, and their derivatives obtained from (31) in (30) we have

$(32)$

$$
\begin{aligned}
& -2 \frac{\partial \varrho}{\partial r}=\frac{\frac{\partial \varphi_{2}}{\partial r} \frac{\partial \varphi_{3}}{\partial t}-\frac{\partial \varphi_{2}}{\partial t} \frac{\partial \varphi_{3}}{\partial r}+2 \varphi_{2} \frac{\partial^{2} \varphi_{3}}{\partial r} \partial t}{\varphi_{2} \frac{\partial \varphi_{3}}{\partial t}}, \\
& -2 \frac{\partial \varrho}{\partial t}=\frac{\frac{\partial \varphi_{1}}{\partial t} \frac{\partial \varphi_{3}}{\partial r}-\frac{\partial \varphi_{1}}{\partial r} \frac{\partial \varphi_{3}}{\partial t}+2 \varphi_{1} \frac{\partial^{2} \varphi_{3}}{\partial r \partial t},}{\varphi_{1} \frac{\partial \varphi_{3}}{\partial r}}, \\
& -\frac{\partial \varrho}{\partial t} \varphi_{2} \frac{\partial \varphi_{3}}{\partial t}-\frac{\partial \varrho}{\partial r} \varphi_{1} \frac{\partial \varphi_{3}}{\partial r}=\varphi_{1} \frac{\partial^{2} \varphi_{3}}{\partial r^{2}}+\varphi_{2} \frac{\partial^{2} \varphi_{33}}{\partial t^{2}},
\end{aligned}
$$

where $\varrho=\log \lambda$. Taking account of (29), these equations may be written

$$
\begin{aligned}
& -2 \frac{\partial \varrho}{\partial r}=\frac{\partial}{\partial r} \log \varphi_{1} \varphi_{2} \varphi_{3}+\frac{2 \varphi_{1} \varphi_{3} G_{1}^{0}}{\frac{\partial \varphi_{3}}{\partial t}}, \\
& -2 \frac{\partial \varrho}{\partial t}=\frac{\partial}{\partial t} \log \varphi_{1} \varphi_{2} \varphi_{3}+\frac{2 \varphi_{1} \varphi_{3} G_{1}^{0}}{\frac{\partial \varphi_{3}}{\partial r}},
\end{aligned}
$$

$\left(3^{\prime}\right) \varphi_{1} \varphi_{2} \varphi_{3}\left(G_{1}^{1}-G_{0}^{0}\right)-\left[\frac{\varphi_{1} \varphi_{3} G_{1}^{0}}{\frac{\partial \varphi_{3}}{\partial t}} \cdot \varphi_{1} \frac{\partial \varphi_{3}}{\partial r}+\frac{\varphi_{1} \varphi_{3} G_{1}^{0}}{\frac{\partial \varphi_{3}}{\partial r}} \cdot \varphi_{2} \frac{\partial \varphi_{3}}{\partial t}\right]=0$ 
The conditions which must be satisfied by the functions $\varphi_{1}, \varphi_{2}$ and $\varphi_{3}$ are therefore, besides $\left(33^{\prime}\right)$, the following:

$$
\frac{\partial}{\partial t}\left[\frac{\varphi_{1} \varphi_{3} G_{1}^{0}}{\frac{\partial \varphi_{3}}{\partial t}}\right]==\frac{\partial}{\partial r}\left[\frac{\varphi_{1} \varphi_{3} G_{1}^{0}}{\frac{\partial \varphi_{3}}{\partial r}}\right] .
$$

We now put

$$
\frac{\varphi_{1} \varphi_{3} G_{1}^{0}}{\frac{\partial \varphi_{3}}{\partial t}}=\frac{\partial \Psi}{\partial r}, \quad \frac{\varphi_{1} \varphi_{3} G_{1}^{0}}{\frac{\partial \varphi_{3}}{\partial r}}=\frac{\partial \Psi}{\partial t},
$$

from which we derive the differential equation for $\Psi$,

$$
\frac{\partial \varphi_{3}}{\partial t} \frac{\partial \Psi}{\partial r}-\frac{\partial \varphi_{3}}{\partial r} \cdot \frac{\partial \Psi}{\partial t}=0 .
$$

Hence, $\Psi s$ must be an arbitrary function of $\varphi_{3}$ or else a constant. The conditions (34) and $\left(33^{\prime}\right)$ may now be written

$$
\begin{aligned}
\varphi_{1} \varphi_{3} G_{1}^{0} & =\Psi^{\prime}\left(\varphi_{3}\right) \frac{\partial \varphi_{3}}{\partial r} \frac{\partial \varphi_{3}}{\partial t} \\
\varphi_{1} \varphi_{2} \varphi_{3}\left(G_{1}^{1}-G_{0}^{0}\right) & =\Psi^{\prime}\left(\varphi_{3}\right)\left[\varphi_{1}\left(\frac{\partial \varphi_{3}}{\partial r}\right)^{2}+\varphi_{2}\left(\frac{\partial \varphi_{3}}{\partial t}\right)^{2}\right] .
\end{aligned}
$$

These conditions being satisfied, the corresponding $G_{1}$ has the form

We have then

$$
U f=-\frac{\frac{\partial \varphi_{3}}{\partial r}}{e^{\Psi} \sqrt{\varphi_{1} \varphi_{2}} \overline{\varphi_{3}}} \cdot \frac{\partial f}{\partial t}+\frac{\frac{\partial \varphi_{3}}{\partial t}}{e^{\Psi}} \frac{\partial f}{\sqrt{\varphi_{1} \varphi_{2} \varphi_{3}}} \cdot \frac{\partial}{\partial r} .
$$

The necessary and sufficient conditions that a centro-symmetric space with linear element

$$
-d s^{2}=\varphi_{2} d r^{2}+\varphi_{3}\left(d \theta^{2}+\sin ^{2} \theta d \varphi^{2}\right)-\varphi_{1} d t^{2},
$$

$\varphi_{1}, \varphi_{2}$ and $\varphi_{3}$ being arbitrary functions of $r$ and $t$ and $\varphi_{3}$ not a constant, shall admit a one-parameter group of motions $G_{1}$ are

$$
\begin{gathered}
\varphi_{1} \varphi_{3} G_{1}^{0}=\Psi \boldsymbol{\nu}^{\prime} \cdot \frac{\partial \varphi_{3}}{\partial r} \frac{\partial \varphi_{3}}{\partial t}, \\
\varphi_{1} \varphi_{2} \varphi_{3}\left(G_{1}^{1}-G_{0}^{0}\right)=+\Psi \boldsymbol{s}^{\prime}\left[\varphi_{1}\left(\frac{\partial \varphi_{3}}{\partial r}\right)^{2}+\varphi_{2}\left(\frac{\partial \varphi_{3}}{\partial t}\right)^{2}\right],
\end{gathered}
$$

where is is an arbitrary function of $y_{3}$, or a constant. 
But we know that by a proper choice of variables this group may always be reduced to the form $\partial f / \partial t$ (Theorem II). The transformation $T=T(r, t)$, $R=R(r, t)$, where $R$ and $T$ satisfy the two partial differential equations

$$
\xi_{0} \frac{\partial T}{\partial t}+\xi_{1} \frac{\partial T}{\partial r}=1, \quad \xi_{0} \frac{\partial R}{\partial t}+\xi_{1} \frac{\partial R}{\partial r}=0
$$

(solved by two quadratures), will carry $\left(21^{\prime}\right)$ into the form

$$
-d s^{2}=\bar{\varphi}_{2} d r^{2}+\bar{\varphi}_{3}\left(d \theta^{2}+\sin ^{2} \theta d \varphi^{2}\right)-\bar{\varphi}_{1} d t^{2},
$$

where $\bar{\varphi}_{1}, \bar{\varphi}_{2}$ and $\bar{\varphi}_{3}$ are functions of $r$ alone. It should be noted that the transformation may always be so chosen as to preserve the orthogonality of the space $S(r, \theta, \varphi)$ to the time-axis. We have therefore

THEOREM V. The necessary and sufficient conditions that a centro-symmetric space $\left(21^{\prime}\right)$ shall be reducible to the static form are given by the equations (35) and $\left(35^{\prime}\right)$.

If we choose the arbitrary function $\boldsymbol{\Psi}=$ const., we have, as a corollary of the above theorem,

If $G_{1}^{1}=G_{0}^{0}$ and $G_{1}^{0}=0$, the centro-symmetric space $\left(21^{\prime}\right)$ is reducible to the static form.

Since for an Einstein solar field we have $G_{i k}=0$ it follows that the Einstein solar field is necessarily static.*

For a class of spaces of importance in relativity theory the generalized Einstein equations hold: $G_{i k}=\lambda g_{i k}$, where $\lambda$ is constant. We have therefore $G_{1}^{0}=0, G_{1}^{1}-G_{0}^{0}=0$. Hence, these spaces are also necessarily static. Examples are De Sitter's cosmological space and the gravitational field of an electron.t

The case $\varphi_{3}=$ const. needs special treatement, since

$$
\frac{\partial \varphi_{3}}{\partial r}=\frac{\partial \varphi_{3}}{\partial t}=0
$$

It will be convenient to transform $\left(21^{\prime}\right)$ into the form

$$
-d s^{2}=\varphi_{1}\left(d r^{2}-d t^{2}\right)+\varphi_{3}\left(d \theta^{2}+\sin ^{2} \theta d \varphi^{2}\right) .
$$

Integrating equations (30) on the hypotheses $\varphi_{1}=\varphi_{2}$ and $\varphi_{3}=$ const. we find

$$
\frac{\partial \xi_{1}}{\partial r}=\frac{\partial \xi_{0}}{\partial t}, \quad \frac{\partial \xi_{1}}{\partial t}=\frac{\partial \xi_{0}}{\partial r}, \quad \varphi_{1}=\varphi_{2}=\varphi\left(r^{2}-t^{2}\right)
$$

* For a different proof of this proposition see G. D. Birkhoff, loc. cit., pp. 253-256.

$\dagger$ A. S. Eddington, The Mathematical Theory of Relativity, Cambridge University Press, 1923, p. 100 and p. 185. 
hence

$$
\xi_{0}=\boldsymbol{\Phi}(r+t)+\Psi \boldsymbol{\Psi}(r-t), \quad \xi_{1}=\boldsymbol{\Phi}(r+t)-\boldsymbol{\Psi}(r-t) .
$$

Without loss of generality we may put $\xi_{1}=t, \xi_{0}=r$, and the group is

$$
U f=r \frac{\partial f}{\partial t}+t \frac{\partial f}{\partial r} .
$$

The integration of (36) will yield the transformation

$$
r+t=R \cdot e^{T}, \quad r-t=R \cdot e^{-T}
$$

which will carry $\left(21^{\prime \prime}\right)$ into the static form. We also find

$$
G_{1}^{0}=0, \quad G_{1}^{1}-G_{0}^{0}=\frac{1}{\varphi_{3}}-\frac{1}{\varphi_{3}}=0 .
$$

To this class belongs the space discussed by Levi-Civita (p. 238, footnote). This space is also necessarily static.

The case where $\varphi_{3}$ is a function of $r$ alone, say $\varphi_{3}=r^{2}$, is not special, since a transformation

will carry $\left(21^{\prime}\right)$ into

$$
R^{2}=\varphi_{3}, \quad T=\Phi(r, t)
$$

$\left(21^{\prime \prime \prime}\right) \quad-d s^{2}=-\bar{\varphi}_{1} d T^{2}+\bar{\varphi}_{2} d R^{2}+R^{2}\left(d \theta^{2}+\sin ^{2} \theta d \varphi^{2}\right)$.

$G_{1}^{0}=0$, since $\partial \varphi_{3} / \partial T=0$ and $\left(34^{\prime}\right)$ shows that $\Psi^{\prime}$ is a function of $R$ alone. The condition $\left(35^{\prime}\right)$ becomes

$$
\bar{\varphi}_{2}\left(G_{1}^{1}-G_{0}^{0}\right)=4 \Psi^{\prime}(R) .
$$

7. We shall suppose that the linear element $\left(21^{\prime}\right)$ admits the groups $G_{1}$ and $G_{3}$, all the transformations of which form a group $G_{4}$, and that it has been reduced to the form (37). If $\varphi_{3}$ is not cor tant, the transformation

$$
R=V \bar{\varphi}_{3}, \quad T=t, \quad \varphi_{2}=1+\bar{\varphi}_{2}
$$

will reduce (37) to the form

$$
d s^{2}=\varphi_{1} d t^{2}-\left(1+\varphi_{2}\right) d r^{2}-r^{2}\left(d \theta^{2}+\sin ^{2} \theta d \varphi^{2}\right)
$$

admitting the group of motions $G_{4}$. 
We have now $h_{i k}=0, i \neq k$, and the mixed tensors $G_{i}^{i}$, which we shall substitute for the tensors $R_{i i}$, become (equations (29))

$$
\begin{gathered}
G_{1}^{1}=\frac{1}{r^{2}\left(1+\varphi_{2}\right)}\left[\varphi_{2}-\frac{r \varphi_{1}^{\prime}}{\varphi_{1}}\right], \quad G_{0}^{0}=\frac{1}{r^{2}\left(1+\varphi_{2}\right)}\left[\varphi_{2}+\frac{r \varphi_{2}^{\prime}}{1+\varphi_{2}}\right] \\
G_{2}^{2}=G_{3}^{3}=\frac{-\varphi_{1}^{\prime \prime}}{2 \varphi_{1}\left(1+\varphi_{2}\right)}+\frac{\varphi_{2}^{\prime} \varphi_{1}^{\prime}}{4 \varphi_{1}\left(1+\varphi_{2}\right)^{2}}+\frac{\varphi_{2}^{\prime}}{2\left(1+\varphi_{2}\right)^{2} r} \\
+\frac{\varphi_{1}^{\prime 2}}{4\left(1+\varphi_{2}\right) \varphi_{1}^{2}}-\frac{\varphi_{1}^{\prime}}{2 \varphi_{1}\left(1+\varphi_{2}\right) r}
\end{gathered}
$$

It appears from these equations that while $G_{1}^{0} \equiv 0$, the same is not true of the difference $G_{1}^{1}-G_{0}^{0}$. We find

$$
G_{1}^{1}-G_{0}^{0}=-\left[\varphi_{1}^{\prime}+\frac{\varphi_{1}}{1+\varphi_{2}} \varphi_{2}^{\prime}\right] \frac{1}{r\left(1+\varphi_{2}\right) \varphi_{1}}
$$

which vanishes if, and only if, $\varphi_{1}\left(1+\varphi_{2}\right)=c$. This ist he "Leithypothese" of Kottler* which may be stated in the form of an equation as follows:

$$
g=\left|\begin{array}{cccc}
g_{11} & g_{12} & g_{13} & 0 \\
g_{12} & g_{22} & g_{23} & 0 \\
g_{13} & g_{23} & g_{33} & 0 \\
0 & 0 & 0 & -g_{00}
\end{array}\right|=\left|\begin{array}{cccc}
a_{11} & a_{12} & a_{13} & 0 \\
a_{12} & a_{22} & a_{23} & 0 \\
a_{13} & a_{23} & a_{33} & 0 \\
0 & 0 & 0 & -c^{2}
\end{array}\right|=\left|a_{i k}\right|\left(-c^{2}\right)
$$

where the $a$ 's are the coefficients of the linear element of a euclidean space and $c$ is the velocity of light in "empty" space. In fact, we have

or

$$
g=-\varphi_{1}\left(1+\varphi_{2}\right) r^{4} \sin ^{2} \theta=r^{4} \sin ^{2} \theta\left(-c^{2}\right),
$$

$$
\varphi_{1}\left(1+\varphi_{2}\right)=c^{2} .
$$

Consider the three sub-spaces $t=c_{1}, y^{\prime}=c_{2}, \theta=c_{3}$, having the respective linear elements

$$
\begin{aligned}
S_{3}: & d s^{2}=\left(1+\varphi_{2}\right) d r^{2}+r^{2}\left(d \theta^{2}+\sin ^{2} \theta d y^{2}\right), \\
(42) \quad S_{3}^{1}: & d s^{2}=\left(1+\varphi_{2}\right) d r^{2}+r^{2} d \theta^{2}-\varphi_{1} d t^{2}, \\
S_{3}^{2}: & d s^{2}=\left(1+\varphi_{2}\right) d r^{2}+r^{2} \sin ^{2} \theta d \varphi^{2}-y_{1}^{\prime} d t^{2} .
\end{aligned}
$$

*F. Kottler, Über die physikalischen Grundlagen der Einsteinschen Gravitationstheorie, Annalen der Physik, vol. 56, pp. 401-462. Kottler considers this hypothesis as a substitute in general relativity for the hypothesis of the constancy of the velocity of light in Minkowski's mechanics. The hypothesis holds for an Einstein solar field, but not necessarily for spaces with a different mass-distribution. It does not hold for Einstein's cosmological space, although it does for that of De Sitter. 
We shall calculate the principal Riemannian curvatures for each of them. We find*

$$
\begin{array}{ll}
S_{3}: & K_{1}=\frac{\varphi_{2}}{r^{2}\left(1+\varphi_{2}\right)}, \quad K_{2}=K_{3}=\frac{\varphi_{2}^{\prime}}{2 r\left(1+\varphi_{2}\right)^{2}}, \\
S_{3}^{1}: & K_{1}^{1}=\frac{-\varphi_{1}^{\prime \prime}}{2 \varphi_{1}\left(1+\varphi_{2}\right)}+\frac{\varphi_{2}^{\prime} \varphi_{1}^{\prime}}{4 \varphi_{1}\left(1+\varphi_{2}\right)^{2}}+\frac{\varphi_{1}^{\prime 2}}{4 \varphi_{1}^{2}\left(1+\varphi_{2}\right)}, \\
& K_{2}^{1}=\frac{\varphi_{1}^{\prime}}{4 \varphi_{1}^{2}\left(1+\varphi_{2}\right)}, \quad K_{3}^{1}=\frac{\varphi_{2}^{\prime}}{2 r\left(1+\varphi_{2}\right)^{2}}, \\
S_{3}^{2}: & K_{1}^{2}=K_{1}, \quad K_{2}^{2}=K_{2}^{1}, \quad K_{3}^{2}=K_{3}^{1}=K_{2}=K_{3} .
\end{array}
$$

Comparing these results with (40) we have, at once,

$$
\begin{aligned}
& G_{0}^{0}=K_{1}+K_{2}+K_{3}, \\
& G_{2}^{2}=G_{3}^{3}=K_{1}^{1}+K_{2}^{1}+K_{3}^{1}=K_{1}^{2}+K_{2}^{2}+K_{3}^{2} .
\end{aligned}
$$

Since in $S_{3}$ we have $K_{2}=K_{3}$, the principal trihedron with respect to any point $P$ has one direction completely determined, namely the direction of the tangent to the $r$-line, while the other two directions through $P$, normal to it and to each other, may be chosen arbitrarily in the tangent plane to the surface $r=$ const. The space $S_{3}$ can therefore rotate freely about the geodesic $r . t$

This is not the case with the spaces $S_{3}^{1}$ and $S_{3}^{2}$, since the three principal curvatures are in general unequal. If, however, $K_{2}^{1}=K_{3}^{1}, S_{3}^{1}$, and also $S_{3}^{2}$ will have the same property as $S_{3}$ : At a point $P$ the principal direction along the $r$-line will be determinate, while the other two are arbitrary in the tangent planes to the surface $r=$ const. The condition $K_{2}^{1}=K_{3}^{1}$ imposes therefore a certain symmetry on the time space $S_{4}$, namely

The three subspaces $S_{3}, S_{3}^{1}, S_{3}^{2}$ possess, at any generic point $P$, "rotational mobility" about the geodesic $r$-line which passes through the point.

It should be noted that, in the case of the subspaces $S_{3}^{1}$ and $S_{3}^{2}$, a "rotation" about the $r$-line means a "hyperbolic" rotation, since these spaces have an indefinite quadratic form as line-element. If with Minkowsky we put $i t=\bar{t}$, the hyperbolic rotation becomes ordinary euclidean.

\footnotetext{
* L. Bianchi, Lezione di Geometria Differenziale, vol. 1, pp. 365-358, and also Lezioni sulla Teoria dei Gruppi, pp. 546-547 by the same author.

$\dagger$ It should be observed in this connection that when we say "rotate freely about the geodesic $r$ " this does not imply that the $r$-line is an axis. It would be a geodesic axis only if $K_{2}=K_{3}=0$, that is, if $\tau_{2}$ is a constant. The transformation $\bar{r}=\int \sqrt{1+\tau_{r}} d r$ will make $\bar{r}$ a geodesic axis.
} 
Since $K_{2}^{1}=K_{3}^{1}$ is equivalent to the condition $G_{1}^{1}=G_{0}^{0}$, or to the condition

$$
\varphi_{1}\left(1+\varphi_{2}\right)=\text { const., }
$$

we see that Kottler's "Leithypothese" is equivalent to the assumption of free mobility of the subspaces $S_{3}, S_{3}^{1}, S_{3}^{2}$ about the $r$-line.

With this assumption the linear element takes the form

$$
-d s^{2}=\left(1+\varphi_{2}\right) d r^{2}+r^{2}\left(d \theta^{2}+\sin ^{2} \theta d \varphi^{2}\right)-\frac{c}{1+\varphi_{2}} d t^{2}
$$

and we also have, from (40),

(47) $G_{1}^{1}=G_{0}^{0}=K_{1}+K_{2}+K_{3}, G_{2}^{2}=G_{3}^{3}=K_{1}^{1}+K_{2}^{1}+K_{3}^{1}=K_{1}^{2}+K_{2}^{2}+K_{3}^{2}$.

8. We shall specialize further by assuming various values for the sum of the three principal curvatures of $S_{3}$, which is the space part of the time-space $S_{4}$.

(a) $K_{1}+K_{2}+K_{3}=0$. We have from (40)

$$
\varphi_{2} \varphi_{1}-r \varphi_{1}^{\prime}=0
$$

or, since $\varphi_{2}=c^{2} / \varphi_{1}-1$,

$$
r \varphi_{1}=c^{2} r+C
$$

Putting $C=-\alpha c^{2}$ we have

$$
\varphi_{1}=c^{2}\left(1-\frac{\alpha}{r}\right), \quad 1+\varphi_{2}=\frac{1}{1-\frac{\alpha}{r}},
$$

which is Schwarzschild's solution for the line-element of a static and stationary space with centro-symmetric mass-distribution, viz.:

$$
-d s^{2}=\frac{d r^{2}}{1-\alpha}+r^{2}\left(d \theta^{2}+\sin ^{2} \theta d \varphi^{2}\right)-r^{2}\left(1-\frac{\alpha}{r}\right) d t^{2} .
$$

This space is characterized by the following three geometrical properties:

1. It admits the group of motions $G_{4}$ (Assumption $L$ ).

2. The three subspaces $S_{1}, S_{2}, S_{3}$ possess rotational mobility about the $r$-line (Assumption $M$ ).

3. The sum of the three principal Riemannian curvatures of the space $S_{3}$ is zero (Assumption $N$ ). 
Assumptions $L, M$, and $N^{*}$ are equivalent to the physical assumption that in the gravitational field outside the mass the tensors. $T_{i k}$ all vanish. In fact Einstein's equations are

$$
G_{i k}=\varkappa T_{i k}=0, \quad \varkappa=\frac{8 \pi k}{c^{4}},
$$

where $k$ is the Newtonian gravitation constant. But from (40) we have

$$
G_{i}^{k}=g^{i k} G_{i k}=0, \quad i \neq k,
$$

and since $\Sigma_{1}^{3} K_{i}=0$ and $K_{2}^{1}=K_{3}^{1}$, it follows that $G_{1}^{1}=G_{0}^{0}=0$. If the values of $\varphi_{1}$ and $1+\varphi_{2}$ are substituted in the expression for $G_{2}^{2}$ and $G_{3}^{3}$ we find that they vanish also.

It will be noted that the principal Riemannian curvatures of the three subspaces $S_{3}, S_{3}^{1}, S_{3}^{2}$ are

$$
K_{1}=K_{1}^{1}=K_{1}^{2}=\frac{\alpha}{r^{3}}, \quad K_{2}=K_{2}^{1}=K_{2}^{2}=K_{3}=K_{3}^{1}=K_{3}^{2}=-\frac{\alpha}{2 r^{3}}
$$

If, as has been suggested by Cesàro,t we put

$$
K=\frac{1}{3} \sum K_{i}, \quad K^{1}=\frac{1}{3} \sum K_{i}^{1}, \quad K^{2}=\frac{1}{3} \sum K_{i}^{2},
$$

anci define $K, K^{1}, K^{2}$ as the mean curvatures of the respective spaces, we have

Theorem VI. The subspaces $S_{3}, S_{3}^{1}$ and $S_{3}^{2}$ of a static time-space (48) with centro-symmetric mass distribution have their mean curvatures equal to zero, and the three principal curvatures of any subspace are respectively equal to the corresponding principal curvatures of any other subspace.

(b) $K_{1}+K_{2}+K_{3}=$ const. $=3 / R^{2}$. We have from (43)

$$
\frac{\varphi_{2} \varphi_{1}-r \varphi_{1}^{\prime}}{r^{2} c^{2}}=\frac{3}{R^{2}}
$$

or

$$
c^{2}-\varphi_{1}-r^{\prime} \varphi_{1}^{\prime}=\frac{3 c^{2} r^{2}}{R^{2}}
$$

${ }^{*} L, M$ and $N$ are also equivalent to postulates $\mathrm{I}-\mathrm{V}$ of Eisenhart's paper The permanent gravilational field in the Einstein theory, Annals of Mathematics, ser. 2, vol.22, No.2; December, 1920.

† Emesto Cesàro, Lezioni di Geometria Intrinsica, Napoli, 1896, p. 223. 
integrating, we have

$$
\varphi_{1}=\frac{C}{r}+c^{2}\left(1-\frac{r^{2}}{R^{2}}\right)
$$

Let $C=0$; the linear element of $S_{4}$ is

$$
-d s^{2}=\frac{d r^{2}}{1-\frac{r^{2}}{R^{2}}}+r^{2}\left(d \theta^{2}+\sin ^{2} \theta d \varphi^{2}\right)-c^{2}\left(1-\frac{r^{2}}{R^{2}}\right) d t^{2} .
$$

This solution applies to the space inside of a homogeneous sphere of matter provided the inertial density (Trägheitsdichte) is assumed to be zero.* If we calculate the Riemannian symbols $(i k, r h)$, we find

$$
(i k, i k)=\frac{1}{R^{2}} a_{i i} a_{k k}, \quad\left(i l_{i}, r h\right)=0, \quad i \neq r, \quad k \neq h,
$$

which means that the curvature $K_{0}=1 / R^{2}$ (L. Bianchi, Lezioni, vol. I, p. 344). The group of $S_{4}$ is therefore the maximum $G_{10}$ of non-euclidean rotations and translations, and that of $S_{3}$ is the corresponding $G_{6}$. $G_{6}$ does not belong to $S_{4}$, while the group $G_{4}$ remains as a subgroup of $G_{10}$. The space (51) is usually referred to as De Sitter's space.

(c) If in (50) we let the constant $C$ differ from zero, we may put $C=c^{2} b^{2} / R^{2}$ and we have

$$
\varphi_{1}=c^{2}\left[1-\frac{r^{2}}{R^{2}}+\frac{b^{3}}{R^{2}} \cdot \frac{1}{r}\right], \quad 1+\varphi_{2}=\frac{1}{1-\frac{r^{2}}{R^{2}}+\frac{b^{3}}{R^{2}} \cdot \frac{1}{r}} .
$$

The space to which this solution applies is that of a shell of thickness $a-b . \quad R$ is supposed to vary between the limits $b>R<a$.t Since the shell acts on the region outside of it like a Newtonian masspoint $m$ determined by the relation

$$
\frac{27 i m}{c^{2}}=\frac{a^{3}-b^{3}}{R^{2}}
$$

${ }^{*}$ H. Weyl, Raum, Zeit und Materie, 4th edition, p. 232; F. Kottler, loc. cit., pp. 438-439. Kottler assumes that the cohesion pressure equals the entire energy of the mass, i. e. $\mu=\varepsilon-p=0$ where $\varepsilon$ is the energy and $p$ the cohesion pressure. See also H.Weyl, loc. cit., p. 254, and p. 256.

†F. Kottler, loc. cit., p. 493. He obtains this solution by assuming that the space has no inertial mass, i. e. the cohesion pressure $p=\varepsilon$. Kottler's solution has not been accepted by Einstein, who prefers the one obtained by Schwarzschild (equation 55). 
we have, for $R>a$,

$$
y_{11}=c^{2}\left[1-\frac{a^{3}-b^{3}}{R^{2}} \frac{1}{r}\right], \quad 1+\varphi_{2}=\frac{1}{1-\frac{a^{3}-b^{3}}{R^{2}} \frac{1}{r}} .
$$

(d) We shall consider the case where $K_{1}+K_{2}+K_{3}$ is inversely proportional to the fourth power of the "distance" $r$ from the mass-center. We have

$$
K=\frac{\varphi_{2} \varphi_{1}-r \varphi_{1}}{r^{2} c^{2}}=\frac{\alpha}{r^{4}}
$$

or, since we are still working under assumption $M$,

Integrating we have

$$
c^{2}-\varphi_{1}-r \varphi_{1}^{\prime}=\frac{c^{2} \alpha}{r^{2}}
$$

$$
\varphi_{1}=c^{2}\left(1-\frac{\beta}{r}+\frac{\alpha}{r^{2}}\right) \text { and } 1+\varphi_{2}=\frac{1}{1-\frac{\beta}{r}+\frac{\alpha}{r^{2}}} .
$$

$S_{4}$ has therefore the linear element

$$
-d s_{2}=\frac{d r^{2}}{1-\frac{\beta}{r}+\frac{\alpha}{r^{2}}}+r^{2}\left(l \theta^{2}+\sin ^{2} \theta d \varphi^{2}\right)-c^{2}\left(1-\frac{\beta}{r}+\frac{\alpha}{r^{2}}\right) d t^{2}
$$

which has been obtained by Weyl.* He considers a sphere having a Newtonian mass $m$ and a static charge $e$. If we put $\beta=2 a$ and $\alpha=k e^{2} / c^{4}$, where $a=k \mathrm{~m} / \mathrm{c}^{2}$, we get the identical form due to Weyl. The group of the space (54) is $G_{4}$, assumptions $L$ and $M$ hold, while for $N$ is substituted (53). This space, and the two preceding ones, belong to a class of spaces characterized by the property of having the sum of the three principal curvatures of $S_{3}$ equal to a function of the distance from the mass-center, assumptions $L$ and $M$ being valid. We find for the value of $\varphi_{1}$

$$
\varphi_{1}=c^{2}\left[1-\frac{1}{r} \int F(r) d r\right]
$$

(e) Let us retain assumption $L$. Instead of $M$, i. e. $K_{2}^{1}=K_{3}^{1}$, we put $M^{\prime}:$

$$
K_{1}^{1}=K_{2}^{1}
$$

* H. Weyl, loc. cit., pp. 236-237. 
and let, as before in case (b),

$$
N^{\prime}: \quad K_{1}+K_{2}+K_{3}=\frac{3}{R^{2}} \text {. }
$$

$\varphi_{1}$ and $\varphi_{2}$ must now satisfy the following differential equations obtained from (42):

$$
\begin{aligned}
\frac{\varphi_{2}}{r^{2}\left(1+\varphi_{2}\right)}+\frac{\varphi_{2}^{\prime}}{r\left(1+\varphi_{2}\right)^{2}} & =\frac{3}{R^{2}}, \\
\frac{\varphi_{1}^{\prime}}{r \varphi_{1}}+\frac{\varphi_{1}^{\prime 2}}{2 \varphi_{1}^{2}}+\frac{\varphi_{2}^{\prime} \varphi_{1}^{\prime}}{2 \varphi_{1}\left(1+\varphi_{2}\right)}-\frac{\varphi_{1}^{\prime \prime}}{\varphi_{1}} & =0 .
\end{aligned}
$$

Integrating the first equation, we find

$$
1+\varphi_{2}=\frac{1}{1-\frac{C}{r}-\frac{r^{2}}{R^{2}}}
$$

we let $C$ be equal to zero and substitute the value of $\varphi_{2}$ in the second equation and integrate; the result is

$$
V_{p_{1}}=c\left[\alpha-\frac{\sqrt{1-\frac{r^{2}}{R^{2}}}}{2}\right],
$$

where $c$ and $\alpha$ are integration constants. If we determine the initial value of $\varphi_{1}$ in such a way that for $r=R$ we have

$$
V_{\varphi_{1}}=\frac{3}{2} c \sqrt{1-\frac{a^{2}}{R^{2}}}
$$

we get

$$
V_{\varphi_{1}}=c\left[\frac{3 \sqrt{1-\frac{a^{2}}{R^{2}}}-\sqrt{1-\frac{r^{2}}{R^{2}}}}{2}\right],
$$

and the linear element of the space $S_{4}$ is

$$
\begin{aligned}
& -d s^{2}=\frac{d r^{2}}{1-\frac{r^{2}}{R^{2}}}+r^{2}\left(d \theta^{2}+\sin ^{2} \theta d y^{2}\right) \\
& -c^{2}\left[\frac{3 \sqrt{1-\frac{a^{2}}{R^{2}}}-\sqrt{1-\frac{r^{2}}{R^{2}}}}{2}\right]_{15^{*}}^{2} d t^{2},
\end{aligned}
$$


which is Schwarzschild's solution for the gravitational space within a liquid sphere of radius $a$. The group of $S_{3}$ is obviously $G_{6}$, but $G_{6}$ does not belong to $S_{4}$. The condition $C=0$ is equivalent to

$$
\text { P: } \quad K_{1}=K_{2}=K_{3}=\frac{1}{R^{2}}
$$

$S_{3}$ is therefore a space of constant curvature. Assumptions $L, M^{\prime}$, and $P$ determine completely the Schwarzschild solution (55).

9. The case $g_{00}=$ const. If $g_{00}$ is constant, the equations (43) become

$$
\begin{gathered}
K_{1}^{1}=K_{2}^{1}=0, \quad K_{1}^{2}=K_{2}^{2}=0, \quad K_{2}=K_{3}=K_{3}^{1}=\frac{\varphi_{2}^{\prime}}{2 r\left(1+\varphi_{22}\right)^{2}}, \\
K_{1}=\frac{\varphi_{2}}{r^{2}\left(1+\varphi_{2}\right)} .
\end{gathered}
$$

The group of $S_{4}$ is the systatic $G_{4}$, and, since $g_{00}$ is constant, the transformation $\partial f / \partial t$ is a translation (Theorem II). The space $S_{4}$ can therefore rotate freely about the $t$-line as geodesic axis. We shall consider the following cases:

(a) $K_{1}+K_{2}+K_{3}=0$. We have

$$
\varphi_{2}\left(1+\varphi_{2}\right)+r \varphi_{2}^{\prime}=0 .
$$

Integrating we find

$$
1+y_{2}=-\frac{1}{1-\frac{\alpha}{r}}
$$

where $\alpha$ is the integration constant. If $\alpha=0$, the space is euclidean. $\alpha \neq 0$ does not correspond to any physical space, since the velocity of light is constant.

(b) $K_{1}+K_{2}+K_{4}=3 / R^{2}$. This case gives us the Einstein-Schrödinger solution for a closed space with incoherent mass in static equilibrium, ${ }^{*}$

$$
-d s^{2}=\frac{d r^{2}}{1-\frac{r^{2}}{R^{2}}}+r^{2}\left(d \theta^{2}+\sin ^{2} \theta d \varphi^{2}\right)-g_{00} d t^{2}
$$

* E. Schrödinger, Über die Lösungssysteme der allgemeinen kovarianten Gravitationsgleichungen, Physikalische Zeitschrift, vol. 19 (1918), p. 20. See also F. Kottler, loc. cit., p. 483, and H. Weyl, loc. cit., pp. 252-253. 
$S_{3}$ is a space of constant curvature $1 / R^{2}$. The group of $S_{4}$ is a $G_{7}$, namely $\partial f / \partial t$, and $G_{6}$ which belongs also to $S_{3} . \quad G_{7}$ is systatic, the systatic spreads being the geodesic $t$-lines.

A general time-space $\left(21^{\prime}\right)$ which admits the group $G_{7}$ can be reduced to the form (56); for since $S_{4}$ admits the group $G_{6}$ which is a transitive group in $S_{3}, \varphi_{1}$ must be constant (Theorem III). Hence, the group $G_{7}$ completely characterizes the Einstein-Schrödinger solution (56).

10. A somewhat interesting type of centro-symmetric spaces is obtained from the form (38) by assuming $\varphi_{3}=$ const. Let this constant be $R^{2}$. The transformation

$$
R \theta=\bar{\theta}, \quad R \varphi=\bar{\varphi}, \quad \sqrt{1+\varphi_{2}} d r=d \bar{r}
$$

will carry the linear element into the form

$$
-d s^{2}=d \bar{r}^{2}+d \bar{\theta}^{2}+\sin ^{2} \frac{\bar{\theta}}{R} d \varphi^{2}-\varphi_{1} d t^{2}
$$

The principal curvatures of the sub-spaces $S_{3}, S_{3}^{1}, S_{3}^{2}$ are

$$
\begin{gathered}
K_{1}=\frac{1}{R^{2}}=G_{1}^{1}=G_{0}^{0} ; \quad K_{2}=K_{3}=0 ; \quad K_{1}^{1}=K_{1}^{2}=0 ; K_{3}^{1}=K_{3}^{2}=0 ; \\
K_{2}^{1}=K_{2}^{2}=-\frac{1}{2 \varphi_{1}} \frac{\partial^{2} \varphi_{1}}{\partial \bar{r}^{2}}+\frac{1}{4 \varphi_{1}^{2}}\left(\frac{\partial \varphi_{1}}{\partial \bar{r}}\right)^{2}=G_{2}^{2}=G_{3}^{3} .
\end{gathered}
$$

$S_{3}$ belongs to a type of $\mathrm{L}$. Bianchi's normal spaces, namely that one for which the three principal curvatures are constant. If the mean curvature $K=\sum K_{i}$ is positive, it is said to be of type $B . *$ This is here the case. The group of the space $S_{4}$ is $G_{4}$ as before, but $S_{3}$ admits a 4-parameter group of motions, namely $G_{3}$ and the translation $\partial f / \partial r$, the latter not belonging to $S_{4}$ except when $\varphi_{1}$ is constant.

A notable case is where the mean curvature of the space $S_{4}$ is zero. We have then

$$
M=\sum_{0}^{3} G_{i}^{i}=\frac{2}{R^{2}}-\frac{1}{\varphi_{1}} \frac{\partial^{2} \varphi_{1}}{\partial \bar{r}^{2}}+\frac{1}{2 \varphi_{1}^{2}}\left(\frac{\partial \varphi_{1}}{\partial \bar{r}}\right)^{2}=0,
$$

which integrated gives

$$
V_{\rho_{1}}=c_{1} e^{\bar{r} / R}+c_{2} e^{-\bar{r}_{r} / R} .
$$

* L. Bianchi, Sugli spazi normali a tre dimenzioni colle curvature principali costanti, Lincei Kendiconti, ser. 5, vol. 25, 1st semester 1916, pp. 59-63. 
This space has been obtained by T. Levi-Civita. He assumes that the physical space $S_{3}$ is supplied with an electrical potential $\varphi=-C r$. The intensity of the force is $|C| \sqrt{g^{11}}$ and is directed normal to the surfaces $r=$ const.* $^{*}$

11. Class of a centro-symmetric space. It follows from a theorem due to E. Kasner that the special Einstein 4-dimensional spread (48) cannot be immersed in a flat 5-space. In other words, the linear element (48) is at least of the second class.t The necessary and sufficient conditions that a differential form $d s^{2}$ in $n$-space shall be of the first class are

1. It must be possible to find a doubly symmetric system of quantities $b_{i k}$ (coefficients of the second differential form) such that

$$
(r k, i h)=b_{r i} b_{k h}-b_{k i} b_{r h}
$$

2. The system $b_{i k}$ must satisfy the differential equations

$$
\left.\frac{\partial b_{r i}}{\partial x_{h}}-\frac{\partial b_{r h}}{\partial x_{i}}+\sum_{t}^{1 \ldots n} \mid r i\right\} b_{h t}-\sum_{t}^{1 \cdots n}\left\{\begin{array}{c}
r \\
t
\end{array}\right\} b_{i t}=0 .
$$

Equations (a) and (b) are known as the generalized equations of Gauss and Codazzi+ for a euclidean $n$-space.

Starting with the general centro-symmetric space $\left(21^{\prime}\right)$ we shall proceed to find the conditions $(a)$. Calculating the Riemannian symbols $(r k, i h)$ we find that they all vanish except the following:

$$
\begin{aligned}
(12,12)= & -\frac{1}{2} \frac{\partial^{2} \varphi_{3}}{\partial r^{2}}+\frac{1}{4 \varphi_{2}} \frac{\partial \varphi_{2}}{\partial r} \frac{\partial \varphi_{3}}{\partial r}+\frac{1}{4 \varphi_{3}}\left(\frac{\partial \varphi_{3}}{\partial r}\right)^{2}+\frac{1}{4 \varphi_{1}} \frac{\partial \varphi_{2}}{\partial t} \frac{\partial \varphi_{3}}{\partial t} \\
(13,13)= & (12,12) \sin ^{2} \theta \\
(10,10)= & -\frac{1}{2} \frac{\partial^{2} \varphi_{2}}{\partial t^{2}}+\frac{1}{2} \frac{\partial^{2} \varphi_{1}}{\partial r^{2}}+\frac{1}{4 \varphi_{2}}\left(\frac{\partial \varphi_{2}}{\partial t}\right)^{2}-\frac{1}{4 \varphi_{2}} \frac{\partial \varphi_{2}}{\partial r} \frac{\partial \varphi_{1}}{\partial r} \\
& -\frac{1}{4 \varphi_{1}}\left(\frac{\partial \varphi_{1}}{\partial r}\right)^{2}+\frac{1}{4 \varphi_{1}} \frac{\partial \varphi_{2}}{\partial t} \frac{\partial \varphi_{1}}{\partial t} \\
(23,23)= & {\left[\varphi_{3}+\frac{1}{4 \varphi_{1}}\left(\frac{\partial \varphi_{3}}{\partial t}\right)^{2}-\frac{1}{4 \varphi_{2}}\left(\frac{\partial \varphi_{3}}{\partial r}\right)^{2}\right] \sin ^{2} \theta }
\end{aligned}
$$

* T. Levi-Civita, Realtà fisica di alcuni spaci normali del Bianchi, Lincei Rendiconti, ser. 5, vol. 25, 1st semester 1917.

†E. Kasner, The impossibility of Einstein fields immersed in a flat space of five dimensions, American Journal of Mathematics, vol. 43, pp. 126-129. For the definition of class see Ricci and Levi-Civita, Méthodes de calcul différentiel absolu, Mathematische Annalen, vol. 54, p. 160.

$\ddagger$ L. Bianchi, Lezioni di Geometria Differenziali, 2d edition, vol. 1, p. 462. 
(58)

$$
\begin{aligned}
(02,02)= & -\frac{1}{2} \frac{\partial^{2} \varphi_{3}}{\partial t^{2}}+\frac{1}{4 \varphi_{2}} \frac{\partial \varphi_{1}}{\partial r} \frac{\partial \varphi_{3}}{\partial r}+\frac{1}{4 \varphi_{3}}\left(\frac{\partial \varphi_{3}}{\partial t}\right)^{2} \\
& +\frac{1}{4 \varphi_{1}} \frac{\partial \varphi_{1}}{\partial t} \frac{\partial \varphi_{3}}{\partial t} ; \\
(03,03)= & (02,02) \sin ^{2} \theta ; \\
(12,02)= & -\frac{1}{2} \frac{\partial^{2} \varphi_{3}}{\partial r \partial t}+\frac{1}{4 \varphi_{2}} \frac{\partial \varphi_{2}}{\partial t} \frac{\partial \varphi_{3}}{\partial r}+\frac{1}{4 \varphi_{3}} \frac{\partial \varphi_{3}}{\partial r} \frac{\partial \varphi_{3}}{\partial t} \\
& +\frac{1}{4 \varphi_{1}} \frac{\partial \varphi_{1}}{\partial r} \frac{\partial \varphi_{3}}{\partial t} ; \\
(13,03)= & (12,02) \sin ^{2} \theta
\end{aligned}
$$

Equations (a) are now

$$
b_{02}=b_{03}=b_{13}=b_{23}=b_{12}=0, \quad b_{01} b_{22}=(12,02),
$$

(59) $b_{01} b_{33}=(13,03), b_{00} b_{33}=(03,03), b_{00} b_{22}=(02,02), b_{11} b_{33}=(13,13)$,

$$
b_{22} b_{33}=(23,23), \quad b_{11} b_{22}=(12,12), \quad b_{00} b_{11}-b_{01}^{2}=(01,01),
$$

from which we derive the relations

$$
\frac{b_{22}}{b_{33}}=\frac{(12,12)}{(13,13)}=\frac{(02,02)}{(03,03)}=\frac{(12,02)}{(13,03)}
$$

that is, we have the following two conditions:

$$
\begin{array}{ll}
\left(a_{1}\right) & (12,12)(03,03)=(02,02)(13,13), \\
\left(a_{2}\right) & (12,12)(13,03)=(13,13)(12,02),
\end{array}
$$

which are satisfied by (58). We also find the following values for the nonvanishing $b$ 's:

$b_{00}^{2}=\frac{(02,02)^{2}}{D}, b_{11}^{2}=\frac{(12,12)^{2}}{D}, b_{22}^{2}=D, b_{33}^{2}=D \sin ^{4} \theta, b_{10}^{2}=\frac{(12,02)^{2}}{D}$,

where $D=(23,23) / \sin ^{2} \theta$. Substituting in the last equation of (59), we obtain the condition

$\left(a_{3}\right)$

$$
(02,02)(13,13)=(01,01)(23,23)+(12,02)(13,03),
$$

which is not satisfied for a general space $\left(21^{\prime}\right)$.

Suppose now that the condition $\left(a_{3}\right)$ is satisfied. A rather long, but not difficult, calculation will show that the $b$ 's also satisfy the Codazzi equations $(b)$. We have thus proved the 
THEOREM VII. A necessary and sufficient condition that the general centrosymmetric space $\left(21^{\prime}\right)$ shall be of the first class is

$\left(a_{3}\right) \quad(02,02)(13,13)=(01,01)(23,23)+(12,02)(13,03)$.

Let the space $\left(21^{\prime}\right)$ be static, and reduced to the form

$$
-d s^{2}=\left(1+\varphi_{2}\right) d r^{2}+r^{2}\left(d \theta^{2}+\sin ^{2} \theta d \varphi^{2}\right)-\varphi_{1} d t^{2}
$$

The condition $\left(a_{3}\right)$ becomes

$$
\varphi_{1}^{\prime \prime}=\frac{1}{2}\left[\frac{\varphi_{1}^{\prime} \varphi_{2}^{\prime}}{\varphi_{2}}+\frac{\left(\varphi_{1}^{\prime}\right)^{2}}{\varphi_{1}}\right],
$$

which integrated gives

$$
\varphi_{2}=\frac{k^{2}}{4} \frac{\left(\varphi_{1}^{\prime}\right)^{2}}{\varphi_{1}}
$$

This condition being satisfied, the spread (38) may be represented in a flat 5-space, the coördinates being

$$
\begin{gathered}
x_{1}=r \sin \theta \sin \varphi, \quad x_{2}=r \sin \theta \cos \varphi, \quad x_{3}=r \cos \theta, \\
x_{4}=k \sqrt{\varphi_{1}} \cos \frac{\bar{t}}{k}, \quad x_{5}=k \sqrt{\varphi_{1}} \sin \frac{\bar{t}}{k},
\end{gathered}
$$

where $\bar{t}=i t$. Hence

$A$ necessary and sufficient condition that the static centro-symmetric space (38) shall be immersible in a flat 5-space is

$$
\varphi_{2}=\frac{k^{2}}{4} \frac{\left(\varphi_{1}^{\prime}\right)^{2}}{\varphi_{1}}
$$

12. Particular spaces which occur in the theory of relativity and for which the condition (60) is satisfied are the following:

1. De Sitter's space (51); $\varphi_{1}=c^{2}\left(1-r^{2} / R^{2}\right), \cdot 1+\varphi_{2}=1 /\left(1-r^{2} / R^{2}\right)$.

2. All spaces for which $g_{00}=\varphi_{1}$ is constant (Einstein's cosmic space).

3. The Schwarzschild solution for a space inside a liquid sphere of radius $a_{0}$ (equation (55)). The coördinates of the 4-spread are

$$
\begin{gathered}
x_{1}=r \sin \theta \sin \varphi, \quad x_{2}=r \sin \theta \cos \varphi, \quad x_{3}=r \cos \theta \\
x_{4}=r\left[3 a_{0}-\sqrt{1-\frac{r^{2}}{R^{2}}}\right] \cos \frac{c \bar{t}}{2 R}, \quad x_{5}=r\left[3 a_{0}-\sqrt{1-\frac{r^{2}}{R^{2}}}\right] \sin \frac{c \bar{t}}{2 R} .
\end{gathered}
$$


This surface is a 4-dimensional torus generated by revolving the 3-dimensional sphere

$$
\left(x_{4}^{2}-3 a_{0} r\right)^{2}+x_{1}^{2}+x_{2}^{2}+x_{3}^{2}=r^{2}
$$

in such a way that the center describes the circle $x_{4}^{2}+x_{5}^{2}=\left(3 a_{0} r\right)^{2}$. The equation of the torus is

$$
\left[\sum_{1}^{3} x_{i}^{2}-r^{2}\left(1+9 a_{0}^{2}\right)\right]^{2}=36 a_{0}^{2} r^{2}\left[r^{2}-\sum_{1}^{3} x_{i}^{2}\right] \text {. }
$$

Since $a_{0}>\frac{1}{3}$, we have $3 a_{0} r>r$ so that the generating sphere does not intersect the $\dot{x}_{1}, x_{2}, x_{3}$ and $x_{4}$-axes. The space inside the liquid sphere in $S_{3}$ is represented by a 3-dimensional spherical cap on the generating sphere defined by the limit $\sum x_{i}^{2}<a_{0}^{2}<r^{2}\left(1-a_{0}^{2}\right)$, and the corresponding time-space by a 4-dimensional zone on the torus generated by the revolution of the cap.

13. We have seen that the space of an Einstein solar field is at least of the second class. We shall prove the following general

THEOREM VIII. The linear element $\left(21^{\prime}\right)$ for which the condition $\left(a_{3}\right)$ is not satisfied is of the second class.

The transformation

$$
\varphi_{3}(r, t)=R^{2}, \quad \psi(r, t)=T
$$

will carry $\left(21^{\prime}\right)$ into the form

$$
-d s^{2}=-\bar{\varphi}_{1} d T^{2}+\bar{\varphi}_{2} d R^{2}+R^{2}\left(d \theta^{2}+\sin ^{2} \theta d \varphi^{2}\right)
$$

or

$$
\begin{aligned}
-d s^{2}=d \sigma^{2}+d s_{0}^{2}=-\bar{\rho}_{1} d T^{2}+ & \left(\bar{\varphi}_{2}-1\right) d R^{2} \\
& +\left(d R^{2}+R^{2} d \theta^{2}+R^{2} \sin ^{2} \theta d \varphi^{2}\right) .
\end{aligned}
$$

Consider the linear element

$$
d \sigma^{2}=-\bar{\varphi}_{1} d T^{2}+\left(\bar{\varphi}_{2}-1\right) d R^{2}
$$

By the general theory of surfaces, if the Gaussian curvature of the 2-spread (62) differs from zero, it is always possible to find three functions $x_{4}, x_{5}$, and $x_{6}$ of $R$ and $T$ such that

$$
d x_{4}^{2}+d x_{5}^{2}+d x_{6}^{2}=d \sigma^{2}
$$

\footnotetext{
${ }^{*} \mathrm{H}$. Weyl, loc. cit., p. 242; $a_{0}=r_{0}$ and $r=a$ in Weyl's notation.
} 
and the linear element (61) may be put in the form

where

$$
-d s^{2}=d x_{1}^{2}+d x_{2}^{2}+d x_{3}^{2}+d x_{4}^{2}+d x_{5}^{2}+d x_{6}^{2},
$$

$$
x_{1}=R \sin \theta \sin \varphi, \quad x_{2}=h \sin \theta \cos \varphi, \quad x_{3}=h \cos \theta .
$$

The 2-spread whose coördinates are $x_{4}, x_{5}$, and $x_{6}$ we shall call the auxiliary surface.

If the Gaussian curvature of the 2-spread (62) is zero, it will be possible to express $d \sigma^{2}$ in the form $d \sigma^{2}=d x_{4}^{2}+d x_{5}^{2}$, that is, the linear element (61) is of the first class. We find

$$
\begin{aligned}
K=\frac{1}{2 V} \frac{1}{\overline{\bar{\varphi}_{1}-\bar{\varphi}_{1}}}=\left\{\frac{\partial}{\partial T}\left(-\frac{1}{\sqrt{\bar{\varphi}_{2}-\bar{\varphi}_{1}}} \cdot \frac{\partial \bar{\varphi}_{2}}{\partial T}\right)\right. \\
\left.+\frac{\partial}{\partial R}\left(\frac{1}{\sqrt{\overline{\bar{\varphi}_{1}-\bar{\varphi}_{1}}}} \cdot \frac{\partial \overline{\bar{\varphi}_{2}}}{\partial R}\right)\right\},
\end{aligned}
$$

or, putting $K$ equal to zero and simplifying,

$$
\begin{aligned}
\frac{\partial^{2} \bar{\varphi}_{1}}{\partial R^{2}}-\frac{\partial^{2} \bar{\varphi}_{2}}{\partial T^{2}}+\frac{1}{2 \bar{\varphi}_{1}} \frac{\partial \bar{\varphi}_{2}}{\partial T} & \frac{\partial \bar{\varphi}_{1}}{\partial T}-\frac{1}{2 \bar{\varphi}_{1}}\left(\frac{\partial \bar{\varphi}_{1}}{\partial R}\right)^{2} \\
& +\frac{1}{2\left(\bar{\varphi}_{2}-1\right)} \cdot\left\{\left(\frac{\partial \bar{\varphi}_{2}}{\partial T}\right)^{2}-\frac{\partial \bar{\varphi}_{2}}{\partial R} \frac{\partial \bar{\varphi}_{1}}{\partial R}\right\}=0 .
\end{aligned}
$$

But this is precisely the condition $\left(a_{3}\right)$, as is easily seen on calculating the requisite Riemannian symbols for the form (61).

In the exceptional case $\varphi_{3}=$ const. no transformation is necessary; $\left(a_{3}\right)$ reduces to $(10,10)=0$, that is, the Gaussian curvature of the 2-space $d \sigma^{2}=-\varphi_{1} d t^{2}+\varphi_{2} d r^{2}$ must be zero. This condition is not satisfied for the space $\left(21^{\prime}\right), \varphi_{3}$ being a constant; it is therefore of the second class. Hence, the space (57) is also of the second class.

A necessary and sufficient condition that a 2-space $(u, v)$ shall be of class zero is that it shall admit an abelian group $\partial f / \partial u, \partial f / \partial v$ as a group of motions. This is the group-property which characterizes all surfaces of zero Gaussian curvature. The condition $\left(a_{3}\right)$ reduces, in the case of the transformed element $\left(21^{\prime \prime \prime}\right)$, to the simpler form

$$
(02,02)(13,13)=(01,01)(23,23),
$$

which by (63) is equivalent to the condition that the sub-space $\varphi=$ const., $\theta= \pm i R+$ const., or the space (62), shall admit an abelian group $G_{2}$ 
of two parameters as a group of motions. Theorems VII and VIII may therefore be stated thus:

A necessary and sufficient condition that the space $\left(21^{\prime \prime \prime}\right)$ shall be of the first class is that the sub-space (62) shall admit an abelian group $G_{2}$ as a group of motions. A 4-space which admits the group $G_{4}$, i. e. the space $\left(21^{\prime}\right)$, is at most of the second class.

A similar statement holds for spaces (21') for which $\varphi_{3}=$ const.

14. Let the space be static and its linear element written in the form

$$
-d s^{2}=d R^{2}+R^{2}\left(d \theta^{2}+\sin ^{2} \theta d \varphi^{2}\right)+\varphi_{2} d R^{2}+\varphi_{1} d T^{2}
$$

where $T=i t$. The spread has the coördinates

$$
\begin{aligned}
& x_{1}=R \sin \theta \sin \varphi, \quad x_{2}=R \sin \theta \cos \varphi, \quad x_{3}=R \cos \theta, \\
& x_{4}=\int \sqrt{\left[\varphi_{2}-\frac{k^{2}}{4} \frac{\left(\varphi_{1}^{\prime}\right)^{2}}{\varphi_{1}}\right]} d R, x_{5}=k \sqrt{\varphi_{1}} \cos \frac{T}{k}, x_{6}=k \sqrt{\rho_{1}} \sin \frac{T}{k} .
\end{aligned}
$$

The auxiliary surface is a surface of revolution generated by revolving the curve

$$
x_{4}=\int \sqrt{\varphi_{2}-\frac{k^{2}\left(\varphi_{1}^{\prime}\right)^{2}}{4 \varphi_{1}}} d h, \quad x_{5}=k V_{\varphi_{1}}^{-}
$$

about the $x_{4}$-axis. The surface $x_{1}=R \sin \varphi, x_{2}=R \cos \varphi, x_{4}=V \overline{\varphi_{2}} d R$ is a generalization of Flamm's quartic surface.*

If in (64) we put $\left(\varphi_{1}^{\prime}\right)^{2}=\left(4 / k^{2}\right) \varphi_{1} \varphi_{2}, x_{4}=0$, and the linear element is of class 1. If we put $\varphi_{2}=\alpha /(R-\alpha), \varphi_{1}=c^{2}(1-\alpha / R)$ and $k=1$, we get the representation of the Einstein solar field in a flat 6-space which was obtained by Kasner in a slightly different form.t

15. Static spaces which are not centro-symmetric are in general of a class higher than 2 . Thus the general static space

$$
-d s^{2}=g_{0} d x_{0}^{2}+g_{1} d x_{1}^{2}+g_{2} d x_{2}^{2}+g_{8} d x_{3}^{2}, \quad x_{0}=i t
$$

${ }^{*}$ H. Weyl, loc. cit., p. 236.

†E. Kasner, Finite representation of the solar gravitational field in a flat space of six dimensions, American Journal of Mathematics, vol. 43, pp. 130-133. It follows as a corollary from Theorem VIII that a general space (21') can be conformally represented on a flat 5-space, and, when $\left(a_{8}\right)$ is satisfied, on a flat 4-space. The space of an Einstein solar field can therefore not be represented conformally on a euclidean 4-space. 
is of the fifth class, or one less than the maximum. To prove this we shall write the element in the form

$$
\left(65^{\prime}\right)-d s^{2}=g_{0}\left[d x_{0}^{2}+\frac{g_{1}}{g_{0}} d x_{1}^{2}+\frac{g_{2}}{g_{0}} d x_{2}^{2}+\frac{g_{3}}{g_{0}} d x_{3}^{2}\right]=g_{0}\left(d x_{0}^{2}+d \sigma^{2}\right) .
$$

But a general curved 3-space may be immersed in a euclidean 6-space so that we have

$$
-d s^{2}=g_{0}\left[d x_{0}^{2}+\sum_{1}^{6} d y_{i}^{2}\right]
$$

the $y$ 's being functions of $x_{1}, x_{2}, x_{3}$. The space (65) is thus represented conformally on a euclidean 7-space. Such a space may be immersed in $a$ euclidean 9-space; for, write

$$
\begin{aligned}
& z_{1}=\sqrt{g_{0}} x_{0}, \quad z_{2}=\sqrt{g_{0}} y_{1}, \cdots, z_{i}=\sqrt{g_{0}} y_{6}, \\
& z_{8}+z_{9}=-\sqrt{g_{0}}, \quad z_{8}-z_{9}=\sqrt{g_{0}}\left(\sum_{1}^{6} y_{i}^{2}+x_{0}^{2}\right),
\end{aligned}
$$

and an easy calculation shows that

$$
-d s^{2}=g_{0}\left(d x_{0}^{2}+\sum_{1}^{6} d y_{i}^{2}\right)=\sum_{1}^{7} d z_{i}^{2}+d z_{8}^{2}-d z_{9}^{2}
$$

Since a 4-space which admits a group $G_{1}$ as complete group of motions can always be reduced to the form (65) it follows that such a space is of the fifth class.

If $\partial f / \partial x_{0}$ is a translation, $g_{0}$ is const. and (65) is of the third class. If, in $\left(65^{\prime}\right)$, the sub-space $d \sigma^{2}$ admits the group $\partial f / \partial x_{3}, g_{1} / g_{0}, g_{2} / g_{0}, g_{3} / g_{0}$ are functions of $x_{1}$ and $x_{2}$ alone. As will be proved in $\$ 16$, a 3-space of this kind is of the first class, i. e. it can be immersed in a euclidean 4-space. (65) is therefore of the third class.

16. Let the space (65) admit the abelian group $\partial f / \partial x_{0}, \partial f / \partial x_{3}$ as complete group of motions, in which case the $g$ 's are functions of $x_{1}$ and $x_{2}$ alone. We shall prove that (65) is of the third class. We write (65) in the form

$$
-d s^{2}=d \sigma^{2}+g_{3} d x_{3}^{2}
$$

$d \sigma^{2}$ is the line-element of a 3 -spread in a euclidean 4-space. To prove this we calculate the Ricmannian symbols, of which the following are non-vanishing:

$$
(10,10), \quad(20,20), \quad(12,12), \quad(10,20),
$$


and set up the Gaussian equations for the calculation of the $b$ 's. We find

$$
\begin{gathered}
b_{00} b_{11}=(10,10), \quad b_{00} b_{22}=(20,20), \quad b_{00} b_{12}=(10,20), \\
b_{11} b_{22}-b_{12}^{2}=(12,12) .
\end{gathered}
$$

These equations determine the $b$ 's and they also satisfy the four Codazzi equations, which we shall not take the trouble of writing here. The lineelement is now

If we put

$$
-d s^{2}==d y_{1}^{2}+d y_{2}^{2}+d y_{3}^{2}+d y_{4}^{2}+y_{3} d x_{3}^{2} .
$$

$$
y_{6}+y_{i}=V \overline{g_{3}}, \quad y_{6}-y_{i}=-x_{3}^{2} V \overline{g_{3}}, \quad y_{5}=V \overline{g_{3}} x_{3},
$$

we have the final result

$$
-d s^{2}==\sum_{i}^{6} d y_{i}^{2}-d y_{\overline{7}}^{2} .
$$

If $\partial f / \partial x_{3}$ is a translation, $g_{3}$ is constant and the 4-spread belongs to a euclidean 5-space. Its class is 1 .

A notable space of this kind is Weyl's cylindrical and static space

$$
-d s^{2}=h\left(d z^{2}+d r^{2}\right)+\frac{r^{2} d \theta^{2}}{f}-f d t^{2},
$$

which is of the third class and admits the group $\partial f / \partial t, \partial f / \partial \theta$ as a complete group of motions.* If $h f=1$ we have the static centro-symmetric space of class 2 which admits a $G_{4}$.

17. Let the 4-space admit the group $\partial f / \partial x_{0}, \partial f / \partial x_{3}, \partial f / \partial x_{2}$; it is found that no reduction in class takes place. The space is of the third class as in the preceding case.

It thus appears that the complete group of a 4 -space (65) determines its class, at least in the case of the abelian group, the group $G_{3}$ of "rotations" and the group of "translations." Whether this is true for all the groups of motions in a 4-space is an open question that might be worth while answering. Fubini's classification of 4-spaces (vols. 8 and 9, Annali di Matematica) would here render a notable service. It should however be noted that the group of certain sub-spaces will also play a rôle in the determination of the class.

*H. Weyl, Annalen der Physik, vol. 54, pp. 134-137.

West Virginia University,

Morgantown, W. Va. 